\title{
Motion of a floating body in a harbour by domain decomposition method
}

\author{
Y.Y. Shi ${ }^{a}$, Z.F. Li ${ }^{\text {b }}$, and G.X. Wu ${ }^{\text {b* }}$ \\ ${ }^{a}$ College of Shipbuilding Engineering, Harbin Engineering University, Harbin 150001, China \\ b School of Naval Architecture and Ocean Engineering, Jiangsu University of Science and \\ Technology, Zhenjiang 212003, China
}

\begin{abstract}
A three-dimensional domain decomposition method is used to solve the problem of wave interaction with a ship floating inside a harbour with arbitrary shape. The linearized velocity potential theory is adopted. The total fluid domain is divided into two sub-ones: one for the harbour and the other for the external open sea. Boundary integral equations together with the free surface Green function are used in the both domains. Matching conditions are imposed on the interface of the two sub-domains to ensure the velocity and pressure continuity. The advantage of the domain decomposition method over the single domain method is that it removes the coastal surface from the boundary integral equation. This subsequently removes the need for elements on the coastal wall when the equation is discretized. The accuracy of the method is demonstrated through convergence study and through the comparison with the published data. Extensive results through the hydrodynamic coefficients, wave exciting forces and ship motions are provided. Highly oscillatory behaviour is observed and its mechanism is discussed. Finally, the effects of incident wave direction, ship location as well as the harbour topography are investigated in detail.
\end{abstract}

Keywords: ship motion in a harbour; domain decomposition; three-dimensional boundary element method; free-surface Green function.

\section{Introduction}

The motion of a ship in a harbour is much more complex than that in the open water, due to the fact that the harbour has its natural frequencies in addition to that of the ship itself, which may lead to a large amplitude motion response of the ship floating inside the harbour. As a result, it may affect cargo handling, or even cause damage to the harbour or the ship itself. Therefore, accurate prediction of the motion behaviour of a ship inside a harbour is of great significance.

There has been a large body of work done on wave motion inside a harbour. Analytical solutions were derived by Mcnown [1] for a circular harbour and by Kravtchenko and Mcnown [2] for a rectangular one. The harbour had a small entrance where the flow into the harbour was

\footnotetext{
* Corresponding author. Permanent address: Department of Mechanical Engineering, University College London, Torrington Place, London WCIE 7JE, UK. Tel.: +44 207679 3870; fax: +44 2073880180.

E-mail address: g.wu@ucl.ac.uk (G.X.Wu)
} 
prescribed. A particular solution was first obtained for the inhomogeneous normal velocity condition at the entrance. This was combined with the general solution satisfying the homogeneous normal velocity condition on the entire harbour boundary including the entrance. In the real problem, the harbour entrance is connected to the external water and flow into the harbour is unknown before the solution is found. Hwang and Tuck [3] studied the harbour wave diffraction problem. The incoming wave to the harbour satisfied the boundary condition on the coastal wall and therefore the wave diffraction was entirely due to the harbour itself. The problem was solved by the boundary integral equation method with sources distributed over both the harbour and coastal walls. The latter was truncated at some distance away from the entrance. Lee [4] divided the fluid domain into the inner harbour region and the external open sea region. This removed the need for the elements on the coastal wall. The solution was obtained by enforcing the flow and pressure continuities at the interface of the two regions. Isaacson and $\mathrm{Qu}[5]$ considered the wave field in a harbour with partially reflecting boundary. Other permeable harbour wall boundary conditions including open, partial reflection and absorbing boundary were used by Hamanaka [6]. A harbour with corner point was considered by Kumar, Zhang [7]. Besides, Martins-Rivas and Mei [8] considered the interaction of waves with a vertical circular cylinder half embedded in cliff and open on the seaside. Although the focus of that work is on the oscillations of the water column inside the cylinder for wave power extraction, the physical problem of which is similar to the wave/harbor interactions. Apart from Laplace equation in the fluid domain or Helmholtz equation in a horizontal plane with the linearized free surface boundary condition, Boussinesq wave model for shallow water was also used to analyze the motion in the harbour [9-12].

In the above work, no floating body is present in the harbour. Sawaragi and Kubo [13] analyzed the motion of a rectangular body floating in a rectangular harbour by domain decomposition method. The whole fluid domain was divided into three sub-domains, a domain of the outer open sea, a domain underneath the ship and the rest of the domain in the harbour. The velocity potential in each domain was expanded into eigenfunctions in the vertical direction. Sawaragi, Aoki [14] proposed a slightly different domain decomposition. Domain one enclosed the ship, domain two covered the rest of the harbour and domain three was the external open sea. The three-dimensional (3-D) boundary element method (BEM) was used in domain one. In domains two and three, eigenfunction expansion was adopted in the vertical direction. The number of terms used in the expansion was balanced by the rate of decay of evanescent modes in the horizontal direction. Takagi, Naito [15] proposed a different domain decomposition method to analyze hydrodynamic force on a floating body in a harbour. The total domain was also divided into three sub-domains. The floating body was enclosed by a cylindrical surface, within which the 3-D BEM was used. 
Outside this cylindrical surface, a finite element method was used for the mild-slope equation up to an external boundary beyond which was the third domain. Ohyama and Tsuchida [16] adopted similar domain decomposition method. The boundary element method was used in a domain surrounding the ship, a mild-slope equation in the outer domain, which was matched with the waves at the far filed. Later Ohyama and Tsuchida [17] solved the problem by including the evanescent modes. Recently, Kumar, Zhang [18] adopted a domain decomposition which was similar to that of Sawaragi, Aoki [14] mentioned previously to investigate the ship motion as well as wave field under the harbour resonance conditions. They adopted the 3-D BEM for the domain surrounding the ship. Away from the ship, the velocity potential was expanded based on eigenfunctions in the vertical dimension in the harbour and open sea. Then the 2-D Helmholtz equation was used for the term of propagating wave in the horizontal plane. For the linear theory different idealizations or approximations have been used to a certain degree, which can be broadly divided into two categories. One is the analytical solution for fluid motion in a harbour of a specific geometry. The other involves domain decomposition. The boundary element method is commonly used near the body together with the series expansion in other domains. However, it is common to ignore the evanescent modes in the expansion or keep only a few terms. In addition to the above work using the linear Stokes wave as the incoming wave, Bingham [19] used the Boussinesq theory for shallow water incoming wave and the body motion was solved in the time domain. However, the nonlinear theory is often adopted for some special wave events. It is not practical to use the fully nonlinear theory to undertake systematic investigations of the general performance of a ship in waves, partly due to the computational effort required.

In this paper, we aim to provide the exact solution in the numerical sense for motion of a ship freely floating inside the harbour and do not introduce any further approximation within the framework of the linearized potential theory. Here 'exact' in the numerical sense means that as the discretization is continuously refined the solution will converge to the exact one. We shall use the 3-D BEM together with the free surface Green function [20]. However when such a method is used directly, the integral equation will involve the coastal wall boundary tending to infinity, on which a huge number of elements may be needed. Therefore, a simpler domain decomposition method to account for wave/harbour interaction [4] is reintroduced in this paper to remove the need of elements along the infinite straight coastal line. The matching condition will be imposed on their interface to enforce flow and pressure continuity condition.

In the next section, the basic theory including mathematical equations and the numerical procedure are first described. In Section 3, to verify the accuracy of the present numerical model and solution, comparisons are made with the published results in a rectangular basin. 
Computations are then carried out for a floating FPSO, and extensive numerical results are provided, including the hydrodynamic forces and ship motions. The highly oscillatory behaviour of the results are observed and its mechanics are detailly discussed. The effect of incident wave frequency and direction, ship location as well as the harbour topography are investigated respectively. Finally, conclusions are drawn in Section 4.

\section{Mathematical model and numerical procedure}

\subsection{Mathematical model}

We consider the problem of motion of a ship floating in a harbour, as sketched in Figure 1. The fluid is assumed to be inviscid and incompressible with constant density $\rho_{0}$, and the flow to be irrotational. The velocity potential theory can then be used. When the amplitudes of the wave and body motions are small compared with the wavelength and characteristic length of the body, the boundary conditions can be linearized, in which all the nonlinear terms can be ignored and the conditions can be imposed on the mean position of the boundary. To describe the problem and develop the numerical procedure, the total fluid domain $\Omega_{A}$ is divided into two sub-domains, the interior domain of harbour $\Omega_{1}$ and the exterior domain of open water $\Omega_{2}$. A Cartesian coordinate system $o-x y z$ is defined in which its origin is on the mean free surface, $y$-axis is along the interface of the two sub-domains and $z$-axis points vertically upwards. The coastal wall along $y$-axis is a straight line and tends to infinity. We also define a body fixed Cartesian coordinate system $O_{g}-x^{\prime} y^{\prime} z^{\prime}$, with its origin at the centre of gravity of the $\operatorname{ship}\left(x_{g}, y_{g}, z_{g}\right), x^{\prime}$ along the ship length, $y^{\prime}$ along the beam and $z^{\prime}$ pointing vertically upwards. The orientation of the ship in the harbour is denoted by the angle $\gamma$ between $x^{\prime}$ and $x$.

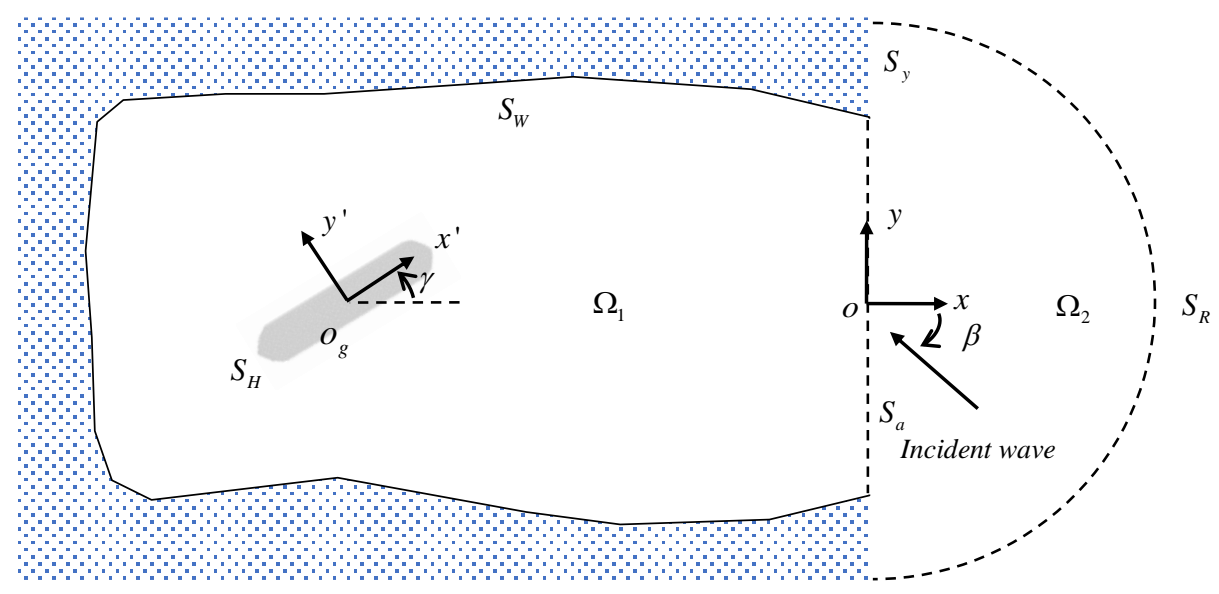

Figure 1 Bird view of a floating ship in a harbour of an arbitrary shape

The velocity potential in each sub-domain can be decomposed into those due to incident, diffraction and radiation 


$$
\Phi^{(l)}(x, y, z, t)=\operatorname{Re}\left\{\left[\phi_{0}^{(l)}(x, y, z)+\sum_{j=1}^{6} \mathrm{i} \omega \eta_{j}^{(l)} \phi_{j}^{(l)}(x, y, z)\right] e^{\mathrm{i} \omega t}\right\},
$$

where periodic motion in time with frequency $\omega$ has been assumed, and $\mathrm{i}=\sqrt{-1}, l=1,2$ correspond to interior and exterior domains, respectively. In Eq. (1), $\phi_{0}^{(l)}$ is the scattering wave potential, $j=1,2,3$ correspond to three translational motions of the body in $x^{\prime}, y^{\prime}, z^{\prime}$ directions, respectively and $j=4,5,6$ are three rotational motions about the axes $x^{\prime}, y^{\prime}, z^{\prime}$ axes respectively, and $\eta_{j}$ is the complex amplitude of the body motion in $j$ mode. $\phi_{j}^{(l)}$ $(j=1, \ldots, 7)$ should satisfy the Laplace equation in the fluid domain

$$
\nabla^{2} \phi_{j}^{(l)}=0
$$

The boundary condition on the free surface $S_{F}$ can be expressed as

$$
\frac{\partial \phi_{j}^{(l)}}{\partial z}-v \phi_{j}^{(l)}=0
$$

where $v=\omega^{2} / g, g$ is acceleration due to gravity. The condition on the fluid bottom $S_{B}$ is

$$
\frac{\partial \phi_{j}^{(l)}}{\partial z}=0
$$

In the interior domain, the impermeable condition on the harbour walls $S_{W}$ is in the form of

$$
\frac{\partial \phi_{j}^{(1)}}{\partial n}=0,(j=0, \ldots, 6),
$$

and the boundary condition on the ship hull surface $S_{H}$ can be expressed as

$$
\frac{\partial \phi_{j}^{(1)}}{\partial n}=n_{j},(j=1, \ldots, 6) \text { and } \frac{\partial \phi_{0}^{(1)}}{\partial n}=0,
$$

where $\vec{n}=\left(n_{x^{\prime}}, n_{y^{\prime}}, n_{z^{\prime}}\right)$ denotes the outward unit normal vector of the surface and

$$
\left(n_{1}, n_{2}, n_{3}\right)=\left(n_{x^{\prime}}, n_{y^{\prime}}, n_{z^{\prime}}\right) \text { and }\left(n_{4}, n_{5}, n_{6}\right)=\left(x^{\prime}, y^{\prime}, z^{\prime}\right) \times\left(n_{x^{\prime}}, n_{y^{\prime}}, n_{z^{\prime}}\right) .
$$

In the exterior domain, the same free surface boundary condition as Eq. (3) should be satisfied. The condition on the coastal wall $S_{y}$, along the $y$ axis and extending to infinity, can be written as

$$
\frac{\partial \phi_{j}^{(2)}}{\partial n}=0,(j=0, \ldots, 6)
$$

The velocity potential should also satisfy the radiation condition on $S_{R}$ at infinity

$$
\lim _{R \rightarrow \infty} \sqrt{R}\left(\frac{\partial \phi_{j}^{(2)}}{\partial R}-i k_{0} \phi_{j}^{(2)}\right)=0,(j=1, \ldots, 6),
$$

to ensure that the wave will propagate outwards, where $R^{2}=x^{2}+y^{2}$, and $k_{0}$ is the purely positive real root of the following dispersion equation

$$
k_{0} \tanh \left(k_{0} h\right)=v .
$$


The radiation condition for the diffraction problem, where $\phi_{7}^{(2)}=\phi_{0}^{(2)}-\phi_{I}$, to ensure the incident wave comes from $\Omega_{2}$, will be discussed later. On the interface $S_{a}$ of domains $\Omega_{1}$ and $\Omega_{2}$ at $x=0$, the following continuity conditions are imposed

$$
\phi_{j}^{1}=\phi_{j}^{2} \text { and } \frac{\partial \phi_{j}^{(1)}}{\partial n}=-\frac{\partial \phi_{j}^{(2)}}{\partial n},(j=0, \ldots, 6)
$$

which is to ensure that the velocity and pressure are continuous. The minus sign in Eq. (11) is due to the fact that $\vec{n}$ is pointing out of the corresponding domain.

\subsection{Numerical procedure}

\subsubsection{Boundary integral equation in the interior harbour domain}

Laplace equation in the fluid domain can be converted into an integral equation over its boundary. Applying the Green's identity in the interior sub-domain $\Omega_{1}$, we have

$$
\alpha^{(1)}(p) \phi_{j}^{(1)}(p)=\int_{S_{W}+S_{H}+S_{a}+S_{F}+S_{B}}\left[G(p, q) \frac{\partial \phi_{j}^{(1)}(q)}{\partial n_{q}}-\frac{\partial G(p, q)}{\partial n_{q}} \phi_{j}^{(1)}(q)\right] d S,(j=0, \ldots, 6),
$$

where $\alpha^{(1)}(p)$ is the solid angle at point $p(x, y, z)$ in $\Omega_{1}$ and the integration is performed with respect to point $q(\xi, \eta, \zeta)$. The Green function $G(p, q)$ adopted in the above equation can be chosen in such a way that it satisfies all the boundary conditions apart from that on the harbour wall, body and interface surfaces. This can be achieved by the free surface Green function in Wehausen and Laitone [20], which can be expressed in the form

$$
G(p, q)=\frac{1}{r}+\frac{1}{r_{2}}+\int_{L} \frac{2(k+v) e^{-k h} \cosh [k(\zeta+h)]}{k \sinh (k h)-v \cosh (k h)} J_{0}(k R) \cosh [k(z+h)] d k,
$$

where $r^{2}=(x-\xi)^{2}+(y-\eta)^{2}+(z-\zeta)^{2}$ is the distance between the field point $p$ and the source point $q, r_{2}^{2}=(x-\xi)^{2}+(y-\eta)^{2}+(z+\zeta+2 h)^{2}, h$ can be taken as the mean water depth of the harbour, $J_{0}(k R)$ refers to the zeroth order Bessel function of the first kind, and the integration route $L$ is from 00 to $\infty$ and should pass over the pole at $k=k_{0}$. We then have

$$
\alpha^{(1)}(p) \phi_{j}^{(1)}(p)=\int_{S_{W}+S_{H}+S_{a}+S_{B}}\left[G^{(1)}(p, q) \frac{\partial \phi_{j}^{(1)}(q)}{\partial n_{q}}-\frac{\partial G^{(1)}(p, q)}{\partial n_{q}} \phi_{j}^{(1)}(q)\right] d S,(j=0, \ldots, 6) .
$$

To take into account the harbour bottom topography effects, we have kept the integration over the seabed $S_{B}$ in Eq. (14). This integral can be removed if the water depth in the harbour is constant $h$.

\subsubsection{Boundary integral equation in the exterior open domain}

Appling the Green's identity in the external domain $\Omega_{2}$, we have 


$$
\alpha^{(2)}(p) \phi_{j}^{(2)}(p)=\int_{S_{y}+S_{a}}\left[G^{(2)}(p, q) \frac{\partial \phi_{j}^{(2)}(q)}{\partial n_{q}}-\frac{\partial G^{(2)}(p, q)}{\partial n_{q}} \phi_{j}^{(2)}(q)\right] d S,(j=1, \ldots, 6),
$$

where $\alpha^{(2)}(p)$ is the solid angle at point $p$ in $\Omega_{2}$. Here the integrations over $S_{F}, S_{B}$ and $S_{R}$ have been removed from Eq. (15), as they are zero when the Green function satisfies conditions on these boundary, together with the potential itself. Direct use of the Green function similar to that in Eq. (12) in Eq. (15) for an arbitrary point in $\Omega_{2}$ will contain the integration over $S_{y}$ in the equation as $G$ does not satisfy the coastal wall condition. To remove such a term, we may modify the Green function as

$$
G^{(2)}=G(p, q)+G(p, \bar{q}),
$$

where $\bar{q}$ is the mirror image of $q$ about $x=0$ plane, which gives $\partial G^{(2)} / \partial n=0$ on $S_{y}$ and $S_{y}$ can be removed from Eq. (15) as a result. $G$ in Eq.(16) has the same form as that in (12), provided the water depth is taken as that in the external domain. When $p$ is, however, on the boundary $S_{y}+S_{a}$, we do have $\partial G / \partial n=0$ itself and Eq. (16) is no longer necessary. Thus,

$$
\alpha^{(2)}\left(p_{a}\right) \phi_{j}^{(2)}\left(p_{a}\right)=\int_{S_{a}} G\left(p_{a}, q\right) \frac{\partial \phi_{j}^{(2)}(q)}{\partial n_{q}} d S,(j=1, \ldots, 6),
$$

where the subscript $a$ indicates $p$ is on $S_{a}$. For $j=0$, we may write the diffraction potential $\phi_{7}^{(2)}$ as

$$
\phi_{7}^{(2)}=\phi_{7}^{(2)^{\prime}}+\phi_{7}^{(2) "},
$$

where

$$
\phi_{7}^{(2) '}(x, y, z)=\phi_{I}(-x, y, z),
$$

which is known directly from the incident potential and is in fact the diffraction potential due to the coastal wall without the harbour, or $S_{y}$ from $y=-\infty$ to $\infty$ completely. Eq. (8) then gives $\partial \phi_{7}^{(2) "} / \partial n=0$ on $S_{y} \cdot \phi_{7}^{(2) "}$ satisfies the radiation condition in Eq. (9). Thus Eq. (17) can be also applied to $\phi_{7}^{(2) "}$.

\subsubsection{Matching at the interface}

To solve the problem numerically, the boundary in each equation is discretized into finite number flat panels, on which the velocity potentials are assumed to be constant. Similar to the method in Hess and Smith [21], Eq. (14) is discretized as

$$
\alpha^{(1)}(p) \phi_{j}^{(1)}(p)=\sum_{m=1}^{N} \int_{S_{m}} \frac{\partial \phi_{j}^{(1)}(q)}{\partial n_{q}} G(p, q) d S_{m}-\sum_{m=1}^{N} \int_{S_{m}} \phi_{j}^{(1)}(q) \frac{\partial G(p, q)}{\partial n_{q}} d S_{m},
$$

where $N$ is the total number of elements, consisting of $N_{H}$ on the floating body, $N_{W}$ on the harbour wall, $N_{B}$ on the non-flat seabed, and $N_{a}$ on the matching surface, and $S_{m}$ is the surface of panel $m$. Here, we may note $N_{W}$ as the summation of $N_{W}$ and $N_{B}$ provided that 
the boundary conditions on them are the same. Similarly, for radiation potential, Eq. (17) can be discretized into

$$
\alpha^{(2)}\left(p_{a}\right) \phi_{j}^{(2)}\left(p_{a}\right)=\sum_{m=1}^{N_{a}} \int_{S_{m}} \frac{\partial \phi_{j}^{(2)}(q)}{\partial n_{q}} G\left(p_{a}, q\right) d S_{m} .
$$

To solve the velocity potentials on all panels simultaneously, Eqs. (20) and (21) can be respectively written in the matrix form

$$
\begin{gathered}
{\left[Q^{(1)}\right]_{N \times N}\left[\phi_{j}^{(1)}(q)\right]_{N}=\left[G^{(1)}\right]_{N \times N}\left[\frac{\partial \phi_{j}^{(1)}(q)}{\partial n_{q}}\right]_{N},} \\
{[Q]_{N_{a} \times N_{a}}\left[\phi_{j}^{(2)}\right]_{N_{a}}=[G]_{N_{a} \times N_{a}}\left[\frac{\partial \phi_{j}^{(2)}}{\partial n}\right]_{N_{a}},}
\end{gathered}
$$

where the matrixes of $[Q]$ and $[G]$ contain the coefficients obtained from the integrations of $\partial G(p, q) / \partial n_{q}$ and $G(p, q)$ over individual panels, respectively. We note that all the coefficients in matrix $[Q]$ in Eq. (23) are zero except the diagonal terms. Using the matching condition in Eq. (11) and then substituting Eq. (23) into (22) we obtain a combined single matrix equation

$$
\left[\begin{array}{lll}
Q_{H H} & Q_{H W} & Q_{H A}+G_{H A} G_{A A}{ }^{-1} Q_{A A} \\
Q_{W H} & Q_{W W} & Q_{W A}+G_{W A} G_{A A}{ }^{-1} Q_{A A} \\
Q_{A H} & Q_{A W} & Q_{A A}+G_{A A} G_{A A}{ }^{-1} Q_{A A}
\end{array}\right]\left[\begin{array}{c}
\phi_{j H}^{(1)} \\
\phi_{j W}^{(1)} \\
\phi_{j A}^{(1)}
\end{array}\right]=\left[\begin{array}{l}
G_{H H} n_{j H} \\
G_{W H} n_{j H} \\
G_{A H} n_{j H}
\end{array}\right],
$$

where the subscripts $H$ and $A$ denote the floating body and interface, respectively, $W$ contains harbour walls and non-flat seabed, and they also imply the corresponding symbols are either sub-matrix or column. For diffraction, the $\phi_{j}^{(2)}$ in Eq. (23) should be replaced by $\phi_{7}^{(2) "}$. Repeating the same procedure, the combined matrix equation can be derived as

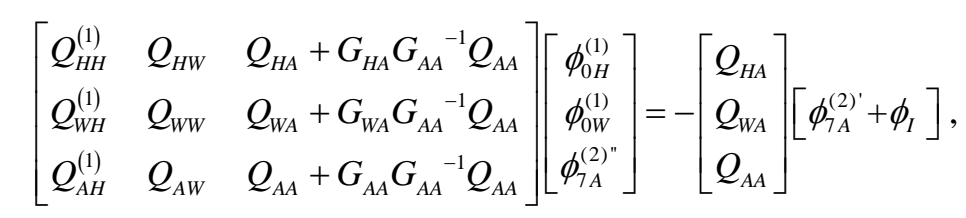

In this way the known valuables are on the right-hand sides of Eqs. (24) and (25), and all the unknowns are on the left hand sides and they can be found simultaneously.

\subsubsection{Hydrodynamic forces and ship motions}

Once the velocity potential is found, the pressure can be obtained through the linearized Bernoulli equation. Then the hydrodynamic force acting on the body can be calculated by integrating the pressure over its wetted surface. The force can be divided into three parts, radiation force coming from the oscillation of the body itself, hydrostatic restoring force caused by the variation of buoyance or the centre of the buoyance and wave excitation force caused by the 
incident and diffracted waves.

The complex motion amplitudes $\eta_{j}$ can be obtained by equations of motions for six degrees of freedom

$$
\sum_{k=1}^{6}\left[-\omega^{2}\left(M_{j k}+A_{j k}\right)-\mathbf{i} \omega B_{j k}+C_{j k}\right] \eta_{k}=f_{j},(j=1, \ldots, 6) .
$$

In Eq. (26), $M_{j k}$ and $C_{j k}$ are the body mass coefficients and the hydrostatic restoring force coefficients, respectively. Their details can be found in [22]. In the present coordinate system, the mass matrix can be written as

$$
[M]=\left[\begin{array}{cccccc}
m & 0 & 0 & 0 & m z_{c} & -m y_{c} \\
0 & m & 0 & -m z_{c} & 0 & m x_{c} \\
0 & 0 & m & m y_{c} & -m x_{c} & 0 \\
0 & -m z_{c} & m y_{c} & I_{22}+I_{33} & -I_{12} & -I_{13} \\
m z_{c} & 0 & -m x_{c} & -I_{12} & I_{11}+I_{33} & -I_{23} \\
-m y_{c} & m x_{c} & 0 & -I_{13} & -I_{23} & I_{11}+I_{33}
\end{array}\right],
$$

where

$$
\begin{gathered}
m=\int_{V} \rho_{b} d v, m x_{c i}=\int_{V} \rho_{b}\left(x_{i}-x_{g i}\right) d v, \\
I_{i j}=\int_{V} \rho_{b}\left(x_{i}-x_{g i}\right)\left(x_{j}-x_{g j}\right) d v \quad(i, j=1, \ldots 3),
\end{gathered}
$$

$\left(x_{1}, x_{2}, x_{3}\right)=(x, y, z)$, and $\rho_{b}$ is density of the body. As the rotational centre is chosen at the centre of gravity, it is obvious that $x_{c}=y_{c}=z_{c}=0$. The restoring coefficients $C_{j k}$ can be written as

$$
\begin{aligned}
& C_{33}=\rho_{0} g A_{w p} ; C_{35}=C_{53}=-\rho_{0} g \int_{w p}\left(x-x_{B}\right) d x d y \\
& C_{44}=\rho_{0} g \nabla\left(z_{B}-z_{g}\right)+\rho_{0} g \int_{w p}\left(y-y_{B}\right)^{2} d x d y, \\
& C_{55}=\rho_{0} g \nabla\left(z_{B}-z_{g}\right)+\rho_{0} g \int_{w p}\left(x-x_{B}\right)^{2} d x d y
\end{aligned}
$$

in which $A_{w p}$ is the waterplane, and $\left(x_{B}, y_{B}, z_{B}\right)$ is the centre of the buoyancy of the body. All the other $C_{i j}$ are equal to zero. The added mass $A_{j k}$, the damping coefficient $B_{j k}$ and the wave excitation force $f_{j}$ can be obtained from

$$
\begin{gathered}
A_{j k}+\frac{B_{j k}}{\mathrm{i} \omega}=\rho_{0} \int_{S_{H}} \phi_{k}^{(1)} n_{j} d S,(j=1, \ldots, 6 ; k=1, \ldots, 6), \\
f_{j}=-\mathrm{i} \rho_{0} \omega \int_{S_{H}} \phi_{0}^{(1)} n_{j} d S,(j=1, \ldots, 6) .
\end{gathered}
$$

\section{Numerical results and discussions}

We first introduce the nondimensionalization. The length of the floating body $L_{s}$, the density 
of fluid $\rho_{0}=1025 \mathrm{~kg} / \mathrm{m}^{3}$ and the gravitational acceleration $g=9.81 \mathrm{~m} / \mathrm{s}^{2}$ are chosen as basic parameters. All the parameters used below are nondimensionalized by their combinations.

\subsection{Convergence study and comparison}

To show the convergence and accuracy of the present methodology, we first consider a rectangular barge inside a harbour. The nondimensionalized length $L_{s}$, width $B_{s}$ and draught $d$ of the body are 1.0, 0.4 and 0.2 respectively. The shape of the harbour is also taken as a rectangle with nondimensionalized length $L_{w}$, width $B_{w}$ and depth $h$ equal to 5.0, 3.0 and 0.5 , respectively. The barge is located at the centre of the harbour and its sides are parallel to those of the harbour. Figure 2 shows the dimensionless added mass and damping coefficients of the barge, in which $\sigma=\omega^{2} L_{s} / g, \quad \mu_{i i}=A_{i i} /\left(\rho L_{s}^{3}\right), \quad \lambda_{i i}=B_{i i} /\left(\rho L_{s}^{3} \cdot \sqrt{g / L_{s}}\right), \quad i=1,2,3, \quad \lambda_{\min }$ is the shortest wave length considered in the figure. $\left(\lambda_{\min } / 32, \lambda_{\min } / 32, \lambda_{\min } / 32\right)$ denote the typical sizes of panels on the floating body, harbour wall and the interface, respectively. It can be seen that very good agreement has been achieved from this mesh and those of $\left(\lambda_{\min } / 44, \lambda_{\min }=44, \lambda_{\min } / 44\right)$, which means that convergence with mesh has been achieved. The sway coefficients are also in good agreement with those in Sawaragi and Kubo [13], taken from Sawaragi, Aoki [14], where an approximation based on a hybrid 3-D and 2-D method was adopted.

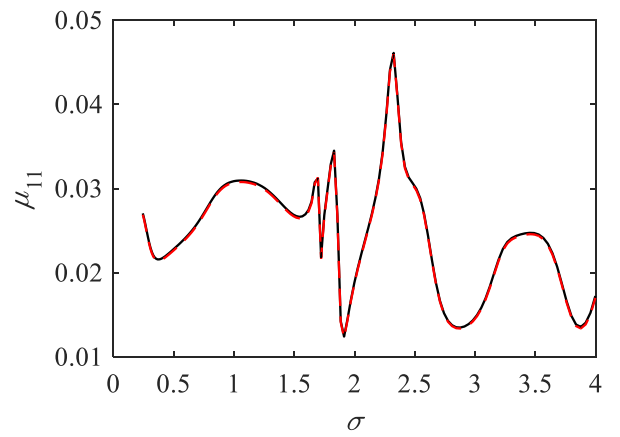

(a)

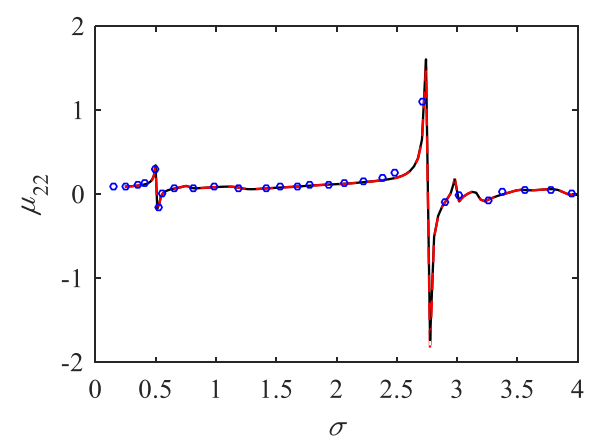

(c)

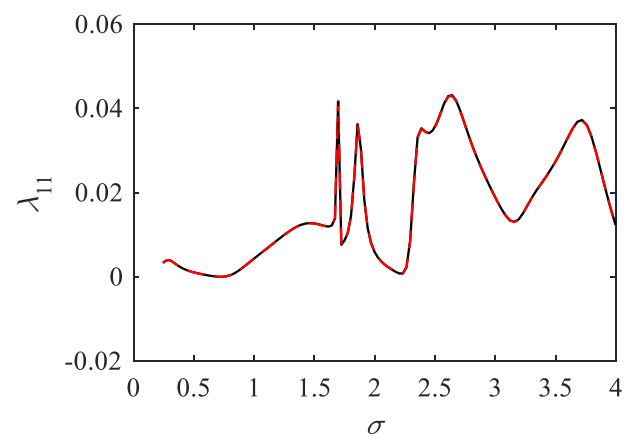

(b)

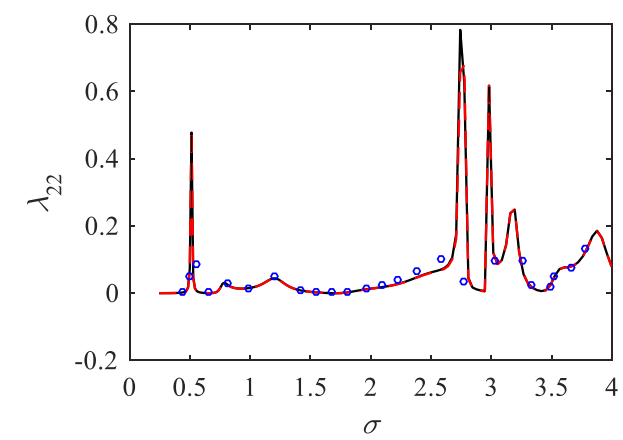

(d) 


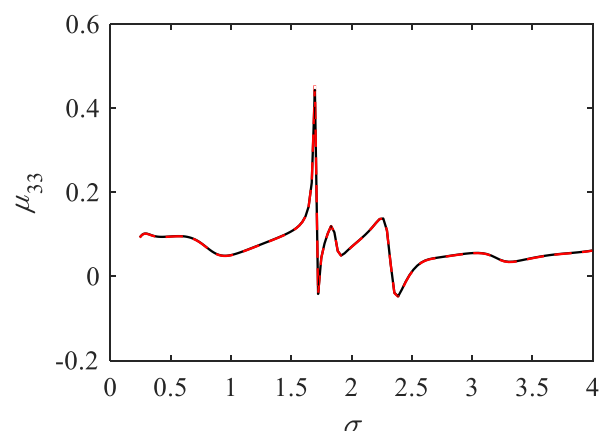

$(e)$

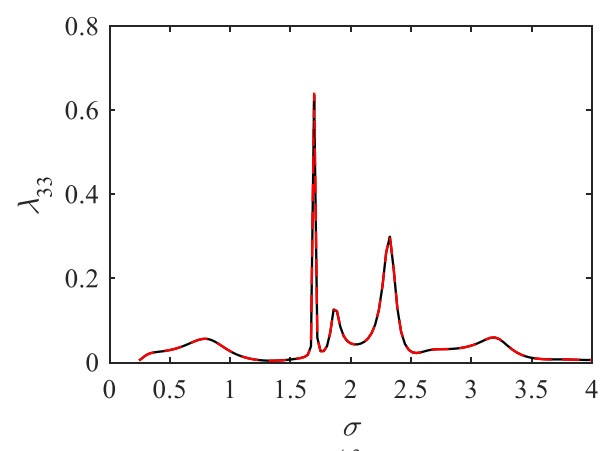

$(f)$

Figure 2 Hydrodynamic coefficients of a barge. Added mass coefficients: (a) surge; (c)sway; (e) heave; Damping coefficients: $(b)$ surge; $(d)$ sway; $(f)$ heave. Solid line: present result with typical element size $\left(\lambda_{\min } / 32, \lambda_{\min } / 32\right.$, $\left.\lambda_{\min } / 32\right)$; dashed line: present result with $\left(\lambda_{\min } / 44, \lambda_{\min } / 44, \lambda_{\min } / 44\right)$, the element area in the second mesh is about the half of that in the first one; open circles: Sawaragi and Kubo (1982)

For scattering problem, the incident velocity potential can be expressed as [22]

$$
\phi_{I}=\frac{\mathrm{i} g \varsigma_{a}}{\omega} \frac{\cosh \left[k_{0}(z+h)\right]}{\cosh \left(k_{0} h\right)} e^{\mathrm{i} k_{0}(x \cos \beta-y \sin \beta)},
$$

where $\beta$ denotes the wave direction shown in Figure 1, and $\varsigma_{a}$ is its amplitude. We consider a scattering problem by a rectangular harbour without the floating body with nondimensionalized length $L_{w}$, width $B_{w}$ and depth $h$ equal to $1,0.1939$ and 0.8268 , respectively, which is based on those in [4]. The amplification factor $R$ is defined as the ratio of $\phi_{0}^{(1)}$ at $\left(-L_{w}, 0,0\right)$ to $\phi_{7}^{\prime}+\phi_{I}$ defined in Eq.(18) at $(0,0,0)$. It can be seen from Figure 3 that the present result is in a good agreement with the analytical solution mentioned in [4].

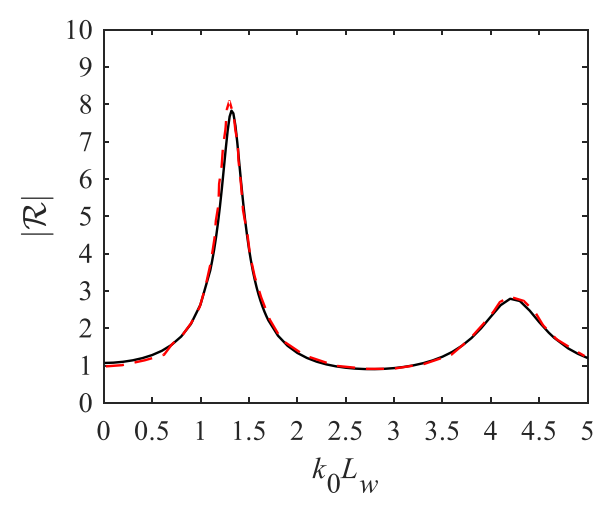

Figure 3 Amplification factor at the centre of the backwall $\left(-L_{w}, 0,0\right)$. Solid line: present result; dashed line: the analytical solution in [4].

A further comparison with the analytical solution Martins-Rivas and Mei [8] for a case which resembles a semi-circular harbour of radius $a$. Instead of fully open to the sea, it is a full vertical cylinder shell with half the body imbedded into the harbour, while the other half is outside the harbour and has an opening from $z=-d$ to the seabed. Figure 4 shows the results for $|\Gamma|^{2}$, 
where $\Gamma$ is the integration of the normal velocity on the free surface inside the cylinder, at $k a=0.901,1.585$ and 2.040 against the incoming wave angle $\alpha(\alpha=\pi / 2-\beta)$, with $a / h=0.5, d / h=0.2$. The element size is taken as $\lambda_{\min } / 39$. It can be seen that results of the present method are in a very good agreement with the analytical solution, which further confirms the accuracy of the present method.

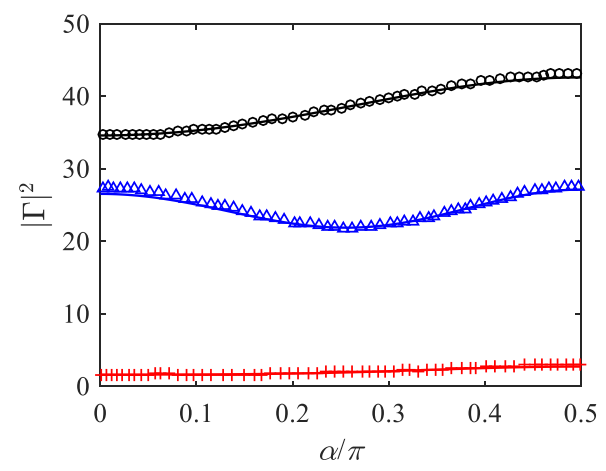

Figure $4|\Gamma|^{2}$ against incoming wave angle for $a / h=0.5, d / h=0.2$. Symbols for analytical solution [8] and the solid lines for the present results. Open circles: $k a=0.901$; open triangles: $k a=1.585$; plus signs: $k a=2.040$.

Then a calculation with the case of the barge $\left(L_{s}=1.0, B_{s}=0.4, d=0.2\right)$ aforementioned floating in the rectangular harbour $\left(L_{w}=5.0, B_{w}=3.0, h=0.5\right)$ is also made with a single domain method. In such a case, elements need to be used over all the coastal walls. In practical computation, the walls at $y>0$ and $y<0$ are truncated at $y=L_{y+}$ and $y=-L_{y-}$ respectively. Figure 5 gives the dimensionless wave exciting force, in which $F_{i}=f_{i} / \rho L_{s}^{3} g, i=1,3$, with $L_{y+} / B_{w}=L_{y-} / B_{w}=1$ and $L_{y+} / B_{w}=L_{y-} / B_{w}=10$, respectively. The element size is taken as $\left(\lambda_{\min } / 32, \lambda_{\min } / 32, \lambda_{\min } / 32\right)$. It can be seen that these two sets of results are quite close to each other, and the results corresponding to $L_{y+} / B_{w}=L_{y-} / B_{w}=10$ are in good agreement with those from the domain decomposition method, although there is still some small difference. This further confirms the effectiveness and accuracy of the present method.

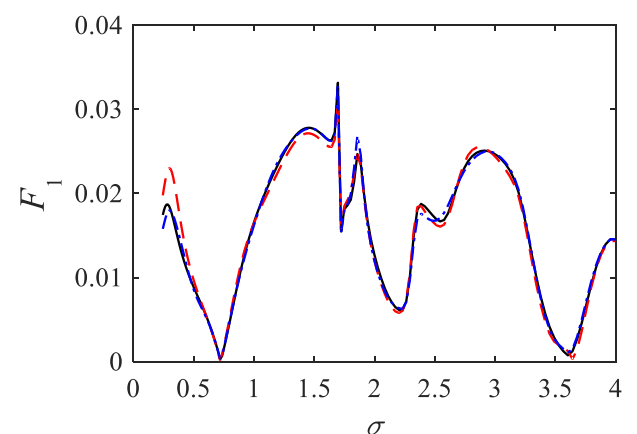

(a)

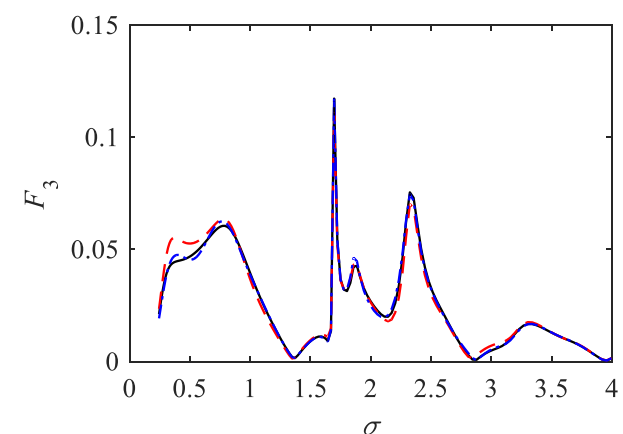

(b)

Figure 5 Excitation forces on a barge $(\beta=0)$ : $(a)$ surge; $(b)$ heave. Solid line: domain decomposition method; dashed line: single domain method with $L_{y+} / B_{w}=L_{y-} / B_{w}=1$; dashed-dotted line: single domain method 
with $L_{y+} / B_{w}=L_{y-} / B_{w}=10$.

\subsection{The oscillatory behaviour of the hydrodynamic coefficients}

In this and the following sections, computations are carried out for a FPSO of uniform weight distribution for the case study. The principal dimensionless data of the ship and the harbour are presented in Table 1 and a sketch of the shape below the waterline is shown in Figure 6.

(a)

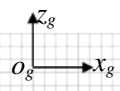

(b)

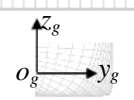

$(c)$

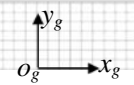

Figure 6 A sketch of FPSO: (a) front view; $(b)$ lateral view; $(c)$ bird view

Table 1 The dimensions of the ship and harbour

\begin{tabular}{|c|c|c|}
\hline Length between perpendiculars & $L_{s}$ & 1.0 \\
\hline Beam & $B_{s}$ & 0.204 \\
\hline Draught & $d$ & 0.071 \\
\hline Gravitational centre & $\left(x_{g}, y_{g}, z_{g}\right)$ & $(-2.5,0.0,-0.054)$ \\
\hline Floatation centre & $\left(x_{B}, y_{B}, z B\right)$ & $(-2.5,0.0,-0.035)$ \\
\hline Inertia radius & $\left(R_{x}, R_{y}, R_{z}\right)$ & $(0.0549,0.0582,0.0582)$ \\
\hline Waterplane area & $A_{w}$ & 0.1905 \\
\hline Transverse metacentre height & $h_{x}$ & 0.0685 \\
\hline Longitudinal metacentre height & $h_{y}$ & 1.1067 \\
\hline Length of harbour & $L_{w}$ & 5 \\
\hline Width of harbour & $B_{w}$ & 2.04 \\
\hline Water depth & $h$ & 0.142 \\
\hline
\end{tabular}

We first consider the radiation problem with $\gamma=0$ and $x^{\prime}=y^{\prime}=0$ is at the centre of the harbour. Calculation is made for $\sigma$ from 0.01 to 4 with step $\Delta \sigma=0.01$. The number of elements on the ship, the harbour and the interface are $N_{H}=1944, N_{w}=2265, N_{a}=385$, respectively, with the ratio of element size to the wavelength is in the range discussed in Section 3.1. Compared with the results in open water, the added mass and damping coefficients in harbour shown in Figure 7 and Figure 8 are quite oscillatory with the frequency. This is not unexpected. In fact, when the harbour is a closed one, or $S_{a}$ becomes a rigid surface, it becomes a rectangular tank with its own natural frequencies which can be obtained from Faltinsen and Timokha [23]

$$
\sigma_{r e s}=k_{r e s} \tanh \left(k_{r e s} h\right)
$$

where

$$
k_{r e s}^{2}=\pi^{2}\left(\frac{m^{2}}{L_{w}^{2}}+\frac{n^{2}}{B_{w}^{2}}\right) \quad(m, n=0,1,2, \ldots) .
$$




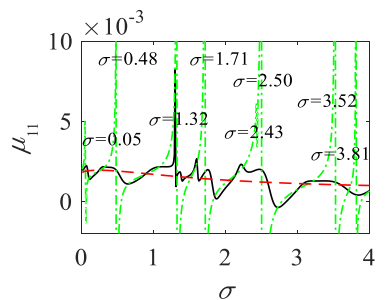

(a)

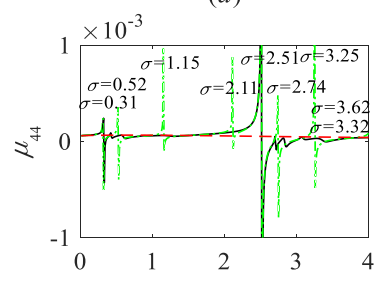

(d)

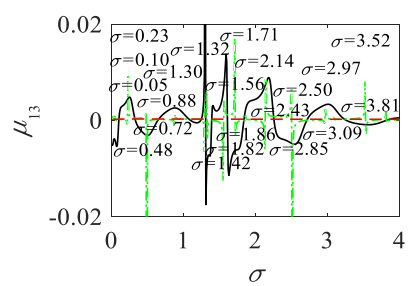

(g)

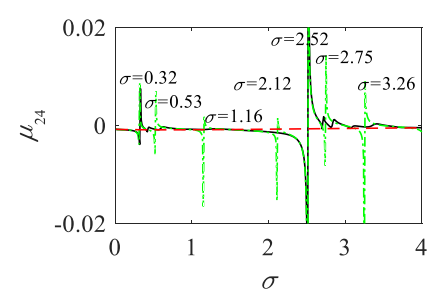

(j)

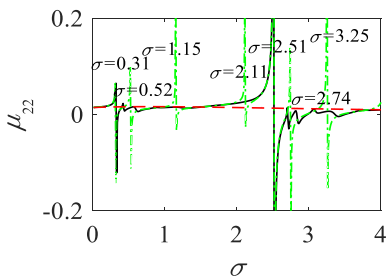

(b)

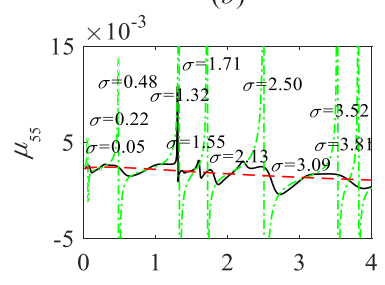

(e)

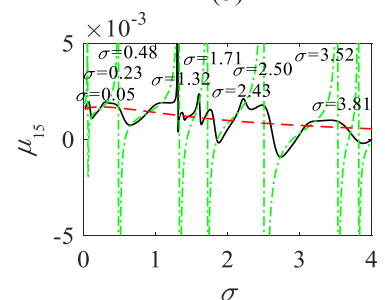

(h)

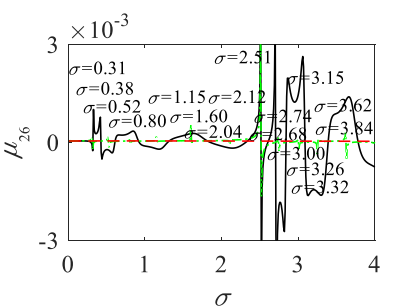

(k)

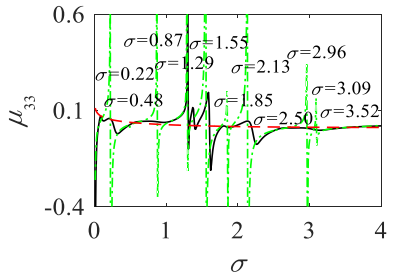

(c)

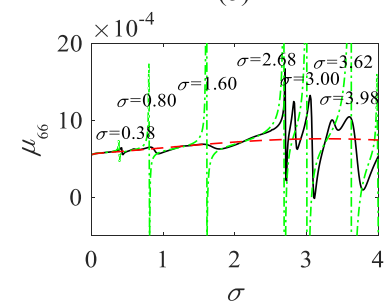

(f)

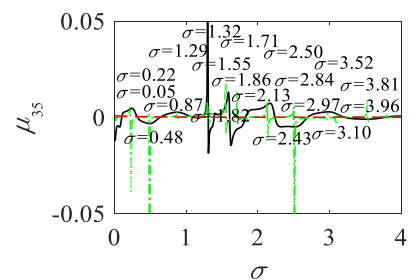

(i)

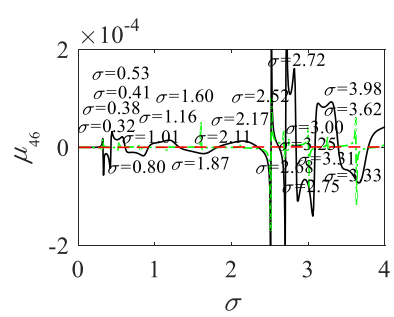

(l)

Figure 7 Added mass coefficients of a FPSO at the centre of the harbour: $(a)$ surge; $(b)$ sway; $(c)$ heave; $(d)$ roll; $(e)$ pitch; $(f)$ yaw; $(g)$ surge- heave; $(h)$ surge- pitch; $(i)$ heave-pitch; $(j)$ sway- roll; $(k)$ sway-yaw; $(l)$ roll- yaw. Solid line: open harbour; dash-dotted line: closed harbour; dashed line: in open water without harbour

We may draw analogy between this and an open harbour with a ship. The results from Eq. (34) with $\sigma<4$, or the upper limit in Figure 7, are shown in Table 2. We also plot the added mass of the ship in the closed harbour in Figure 7, while the damping coefficient is zero as there is no wave propagating out. The natural frequencies of a closed harbour with and without the presence of the ship will not be exactly the same. However, when dimension of the ship is relatively small compared with that of the harbour, we expect these two sets of natural frequencies to be close. It should be noted that even and odd $m$ in Eq. (35) respectively correspond to flows symmetric and anti-symmetric about the $x^{\prime}=0$ plane, and similarly $n$ about the $y^{\prime}=0$ plane. As the problem with a ship is symmetric about the plane $y^{\prime}=0$, flows due to surge, heave and pitch will be symmetric, while the flows due to sway, roll and yaw will be anti-symmetric. The ship does not have fully fore and aft symmetry. However, the large middle section is symmetric about $\quad x^{\prime}=0$ 
and approximate symmetry and anti-symmetry can be applied too. For this reason, the locations of peaks, corresponding to the added mass in the closed harbour can be closely linked to the results in Table 2 at the corresponding even or odd $m$ and $n$. Coupled resonance can also been indicated in Figure 7 , at $\sigma=0.22,0.48 \ldots$ in heave and pitch. For a ship in an open harbour, the natural frequencies are not expected to be the same as those in the closed harbour. It can be seen that there may be a less number of large peaks in the open harbour, but when a small peak occurs its location is very close to one of the natural frequencies in the closed harbour.

When resonance occurs in a closed harbour, the wave amplitude tends infinity based on the linear theory. This is reflected by the added mass in Figure 7. For an open harbour, the wave amplitude and added mass will not tend to infinity because there will be damping due to wave radiation into infinity. However, in both cases, we observe the added mass change very rapidly near the resonance and it can even be negative. It is well known that the diagonal added mass in the unbounded fluid is definitely positive as it is related to the kinetic energy of fluid flow [24]. Here from Eq. (31), we have

$$
A_{i j}=\frac{1}{2} \rho_{0} \int_{S_{H}}\left(\phi_{j} n_{i}+\phi_{j}^{*} n_{i}\right) d S
$$

where * indicates the complex conjugate. Replacing second and first $n_{i}$ in the above equation with the normal derivative in Eq. (6) and its conjugate respectively, and using the Green identity in the entirely fluid domain, we have

$$
A_{i j}=\rho_{0} \operatorname{Re}\left[\int_{\Omega}\left(\nabla \phi_{j} \cdot \nabla \phi_{i}\right) d V\right]-\rho_{0} g \operatorname{Re}\left[\int_{S_{F}} \eta_{j} \eta_{i} d S\right],
$$

where the free surface and radiation conditions have been used. This shows that the added mass is due to the difference between the kinetic energy and the potential energy. Thus, there is no guarantee that the added mass will be positive. Near resonance, both terms in Eq. (37) will become very large, and they are in fact infinite at resonance of a closed harbour. Thus, a relatively small variation in each term could lead to completely different total result. This is reflected by the sharp change in Figure 7, from a large positive value to large negative one in some cases, near the resonance.

The non-diagonal terms such as $\mu_{13}=\mu_{31}, \mu_{15}=\mu_{51}$ have been given in Figure 7(g)-(l). Different from those in open water, these coupling terms are significantly influenced by the natural frequencies of the harbour. The positions of peaks of the $\mu_{13}$ are virtually a combination of those for $\mu_{11}$ and $\mu_{33}$. The magnitudes of coupling terms are much larger than those in open water.

Table 2 The natural frequencies of the closed harbour (Eqs.(34) and (35)) 


\begin{tabular}{llllll}
\hline$n$ & $m$ & $\sigma_{\text {res }}$ & $n$ & $m$ & $\sigma_{\text {res }}$ \\
0 & 0 & 0.00 & 1 & 7 & 2.70 \\
0 & 1 & 0.06 & 1 & 8 & 3.33 \\
0 & 2 & 0.22 & 1 & 9 & 4.00 \\
0 & 3 & 0.49 & 2 & 0 & 1.26 \\
0 & 4 & 0.86 & 2 & 1.31 \\
0 & 5 & 1.32 & 2 & 1.46 \\
0 & 6 & 1.85 & 2 & 1.70 \\
0 & 7 & 2.44 & 2 & 2.03 \\
0 & 8 & 3.08 & 2 & 4 & 2.44 \\
0 & 9 & 3.77 & 2 & 5 & 2.91 \\
1 & 0 & 0.33 & 3 & 3.45 \\
1 & 1 & 0.38 & 3 & 7 & 2.65 \\
1 & 2 & 0.55 & 3 & 0 & 2.70 \\
1 & 3 & 3 & 1 & 2.83 \\
1 & 4 & 0.81 & 3 & 2 & 3.04 \\
1 & 5 & 1.17 & 3 & 5 & 3.32 \\
1 & 5 & 2.12 & 51 & 5 & 3 \\
\hline
\end{tabular}

While the added mass is linked to the difference between the kinetic energy and the potential energy, the diagonal damping coefficient depends on the wave radiation energy and it is always positive, which is shown in Figure 8. Generally, it can be seen from Figure 7 and Figure 8 that the positions of damping coefficient peaks are close to those of added mass peaks and trough. The former occurs for example at $\sigma=1.30$ for surge, heave and pitch of symmetric modes, while the latter occurs in anti-symmetric modes at $\sigma=2.71$ in sway, roll and yaw, and also at $\sigma=0.33$, 2.52 in sway and roll.

The behaviour of the hydrodynamic coefficients can be further explained through the method based on wide spacing approximation. We may follow the practice of the strip theory and consider a transverse cross section of the ship. Based on wide spacing approximation the added mass and damping coefficient of this section can be written as [25]

$$
A_{j k}-\mathrm{i} \frac{B_{j k}}{\omega}=A_{j k}^{o}-\mathrm{i} \frac{B_{j k}^{o}}{\omega}-\varepsilon_{k}^{1} \frac{f_{j}^{o+}}{g}-\varepsilon_{k}^{2} \frac{f_{j}^{o-}}{g},
$$

where the superscript o indicates the result from the open water, and $f_{k}$ is the exciting force. In the above equation 


$$
\begin{aligned}
& \varepsilon_{k}^{1}=-\left[\left(\wp_{k}^{-} t_{0}^{-}-\wp_{k}^{+} r_{0}^{-}\right) \mathcal{R}^{2} e^{-\mathrm{i} k_{0} B_{w}}+\wp_{k}^{+} \mathcal{R}\right] / \mathcal{M} \\
& \varepsilon_{k}^{2}=-\left[\left(\wp_{k}^{+} t_{0}^{+}-\wp_{k}^{-} r_{0}^{+}\right) \mathcal{R}^{2} e^{-\mathrm{i} k_{0} B_{w}}+\wp_{k}^{-} \mathcal{R}\right] / \mathcal{M} \\
& \eta_{k}^{1}=-\left[\left(\wp_{k}^{+} t_{0}^{+}-\wp_{k}^{-} r_{0}^{+}\right) \mathcal{R} e^{-\frac{1}{2} \mathrm{i}_{0} B_{w}}+\wp_{k}^{-} e^{\frac{1}{2} k_{0} B_{w}}\right] / \mathcal{M}, \\
& \eta_{k}^{2}=-\left[\left(\wp_{k}^{-} t_{0}^{-}-\wp_{k}^{+} r_{0}^{-}\right) \mathcal{R} e^{-\frac{1}{2} k_{0} B_{w}}+\wp_{k}^{+} e^{\frac{1}{2} k_{0} B_{w}}\right] / \mathcal{M}
\end{aligned}
$$

where

$$
\mathcal{M}=\left(t_{0}^{+} t_{0}^{-}-r_{0}^{+} r_{0}^{-}\right) \mathcal{R}^{2} e^{-\mathrm{i} k_{0} B_{w}}-e^{\mathrm{i} k_{0} B_{w}}+r_{0}^{-} \mathcal{R}+r_{0}^{+} \mathcal{R} .
$$

Here $\wp_{k}^{ \pm}$is the amplitude of radiation potential at $y \rightarrow \pm \infty$ due to the forced body oscillation in the k-th mode with unit amplitude; $r_{0}^{ \pm}$and $t_{0}^{ \pm}$are respectively the reflection and transmission coefficients for the incident wave from $y \rightarrow \pm \infty ; \mathcal{R}$ and $\mathcal{T}$ are respectively the reflection and transmission coefficients by the harbour wall, and therefore $\mathcal{R}=1$ and $\mathcal{T}=0$. As $\sigma$ increases, $t_{0}^{ \pm}=t \rightarrow 0, \quad r_{0}^{ \pm}=r$ and $|r| \rightarrow 1$ [26]. we have

$$
\varepsilon_{k}^{2}=\frac{\wp_{k}^{-}}{e^{k_{0} l}-r}
$$

while $\varepsilon_{k}^{1}=(-1)^{k} \varepsilon_{k}^{2}, f_{k}^{+}=(-1)^{k} f_{k}^{-}$.

The far field formula for $f_{j}^{0-}$ can be given as

$$
f_{j}^{\mathrm{o}-}=-2 \mathrm{i} \rho \omega \wp_{j}^{-} C_{g},
$$

where $C_{g}$ is the wave group velocity in the open water. Substituting Eqs. (41) and (42) into Eq.(38), we have

$$
\begin{aligned}
& A_{j k}=A_{j k}^{\mathrm{o}}-\frac{2 \rho \omega C_{g}\left[1+(-1)^{j+k}\right]}{g} \operatorname{Im}\left(\mathcal{A}_{j k}\right), \\
& B_{j k}=B_{j k}^{\mathrm{o}}-\frac{2 \rho \omega^{2} C_{g}\left[1+(-1)^{j+k}\right]}{g} \operatorname{Re}\left(\mathcal{A}_{j k}\right),
\end{aligned}
$$

where

$$
\mathcal{A}_{j k}=\frac{\wp_{j}^{-} \wp_{k}^{-}}{\mathrm{e}^{k_{0} l}-r} .
$$

Thus Eq. (45) can be written as

$$
\mathcal{A}_{j k}=-\frac{\left|\wp_{j}^{-} \wp_{k}^{-}\right|}{e^{\mathrm{i}\left(k_{0} B_{w}-\arg r\right)}-|r|} e^{\mathrm{i}\left[\arg \left(\wp_{j}^{-}\right)+\arg \left(\wp_{j}^{-}\right)-\arg (r)\right]} .
$$

The denominator of Eq. (46) can be very small when $k_{0} B_{w}-\arg r=2 n \pi$. Therefore by substituting Eq. (46) into (43), we can see that a sharp jump from a positive peak to negative trough will appear when $|r| \rightarrow 1$ based on this wide harbour approximation. We also note that the condition of $k_{0} B_{w}-\arg r=2 n \pi$ is merely dependent on the shape of the section. Since the cross 
section of the FPSO does not change very much over a large part along the length, the equation may be used for the whole ship. It is interesting to see that this condition is in fact consistent with Eq. (35), where $m=0$ should be taken as the above approximation has ignored wall at $x=-L_{w}$. It also should be noted that $\arg (r)$ may affect the exact location of each point but it does not affect the distribution of the points.

The damping coefficients in an open harbour can also be found from the far-field method [26], which may be modified for the present problem with a half circular control surface at infinity. The solution on the body was finally switched on the surface of the open entrance. It can be seen in Figure 8 that the far field equation gives the same results as the near-field method, apart from a small difference when the damping changes sharply with the frequency. That once again confirms the accuracy of the present method.

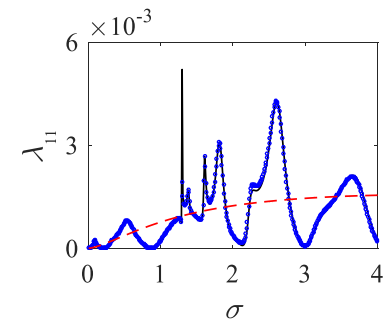

(a)

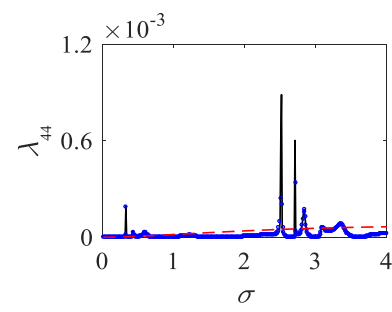

(d)

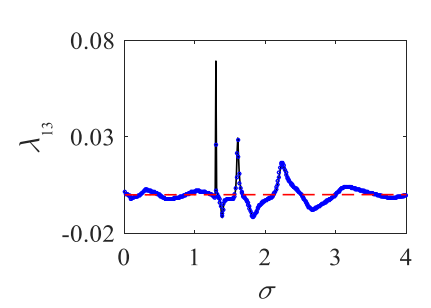

(g)

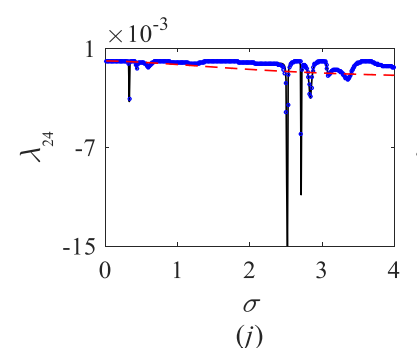

(j)

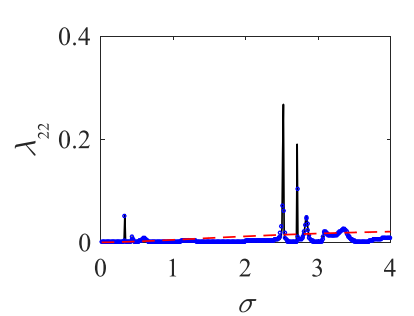

(b)

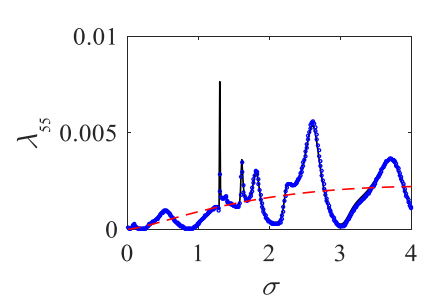

(e)

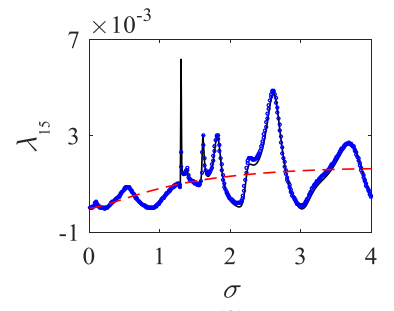

(h)

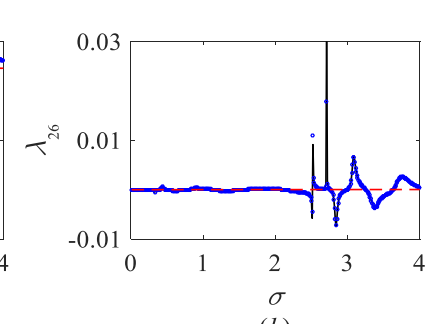

(k)

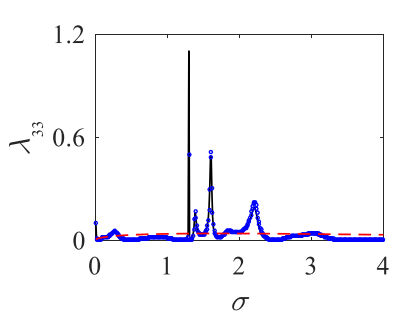

(c)

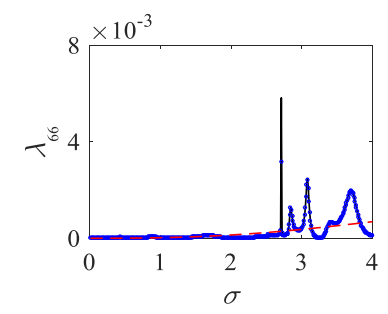

(f)

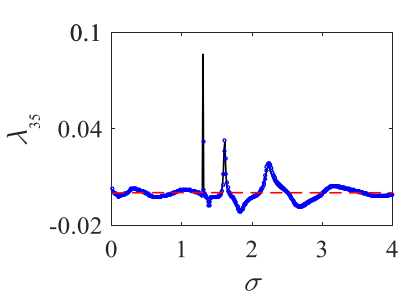

(i)

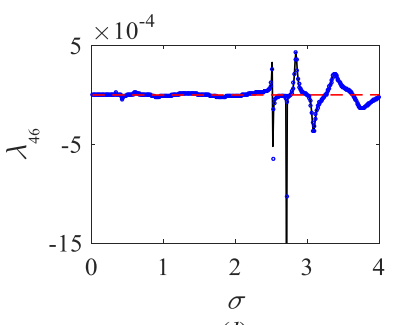

(l)

Figure 8 The same as Figure 7 but for damping coefficients: $(a)$ surge; $(b)$ sway; $(c)$ heave; $(d)$ roll; $(e)$ pitch; $(f)$ yaw; $(g)$ surge- heave; $(h)$ surge- pitch; $(i)$ heave-pitch; $(j)$ sway- roll; $(k)$ sway-yaw; $(l)$ roll- yaw. Solid line: open 
harbour; open circles: open harbour with far field method; dashed line: in open water without harbour

\subsection{The effect of incident wave direction}

The exciting force due to incident wave of angle $\beta$ varying in range of $[0, \pi / 2]$ is considered next, with step $\Delta \beta=\pi / 8$ and the wave amplitude taken as unit. Results for $\beta<0$ is omitted because of symmetry. In the open water, the excitation force of surge or pitch motion is usually the largest at the head sea $(\beta=0)$ and smallest at the beam sea $(\beta=\pi / 2)$. In fact, in the latter case, excitation in surge, pitch and yaw would be zero if the FPSO were of fore and after symmetry. Figure 6 shows that the FPSO has small asymmetry between the bow and stern. Thus, the nondimensional forces $F_{1}, F_{5}$ and $F_{6}$ will be expected to be close to zero in the open sea at $\beta=\pi / 2$. For roll motion, the loading in the beam sea is usually larger than that in quartering sea $(\beta=\pi / 4)$, while it is zero in the head sea in the open sea. In the open harbour, these situations, however, are quite different. For the surge or pitch motion, the force at $\beta \neq 0$ can be larger than that in the head sea in some frequencies while for the roll motion the excitation in quartering sea can be larger than that of the beam sea in some ranges of frequencies. This is once again partly due to the natural modes of the harbour. As several natural frequencies are close to each other, complex wave pattern can appear, against the radially propagating wave pattern in the open sea.

The excitation force also exhibits the highly oscillatory behaviour with the frequency. This is similar to the behaviour of hydrodynamic coefficients but not identical. If we consider the case of $\beta=0$, the problem can be approximated by using a mirror image about the inner wall of the harbour at $x=-L_{w}$. Then at certain frequencies, the excitation due to incident waves from $x=\infty$ and $x=-\infty$ will have strong cancellation. This is reflected by the results of $F_{1}, F_{3}$ and $F_{5}$ in Figure 9. The similar phenomenon can be also seen in [27] in which a breakwater is floating inside an open harbour. The excitations in other modes remain zero due to symmetry. A large peak occurs at $\sigma=1.30$ and $\sigma=2.52$ in symmetric modes and anti-symmetric modes of the natural frequency respectively, which is obviously due to harbour resonance, as same as that in Figure 7 and Figure 8.

As the damping coefficient, the exciting force on the ship may also be found by the far-field method [26]. They are used to check the results in Figure 9 and a good agreement can be found. While $\sigma \rightarrow 0$, according to the far field expression the force on the ship in the harbour can be simplified as

$$
f_{j}(\sigma \rightarrow 0)=2 \rho_{0} g \varsigma_{a} \int_{S_{a}} \frac{\partial \phi_{j}}{\partial n} d s
$$

Using divergence theorem on all boundaries in the interior domain $\Omega_{1}$ and applying boundary conditions we obtain 


$$
f_{j}(\sigma \rightarrow 0)=-2 \rho_{0} g \varsigma_{a} \int_{S_{H}} \frac{\partial \phi_{j}}{\partial n} d s=-2 \rho_{0} g \varsigma_{a} \int_{S_{H}} n_{j} d s=2 \rho_{0} g \varsigma_{a} \int_{S_{w p}} n_{j} d s
$$

where $S_{w p}$ is the surface of the waterplane. This result is independent of wave direction. For $j=3$, the integration in Eq. (48) is $A_{w p} L_{s}^{2}$ while it is zero for $j=2,4,6$ and close to zero for $j=1,5$ as the ship is of slight fore and aft asymmetry. To investigate the behaviour of the result when $\sigma \rightarrow 0$ closely, calculation is made up to $\sigma=0.0001$ in Figure 9. We notice that $F_{3}=\left|f_{3} /\left(\rho_{0} g L_{s}^{3}\right)\right|=0.00165$ at that frequency, and thus $\left|f_{3} /\left(\rho_{0} g \zeta_{a} A_{w p} L_{s}^{2}\right)\right|=1.95$, which is close to 2 . This verifies Eq. (48). However, we also notice that the result changes very rapidly at small frequency.

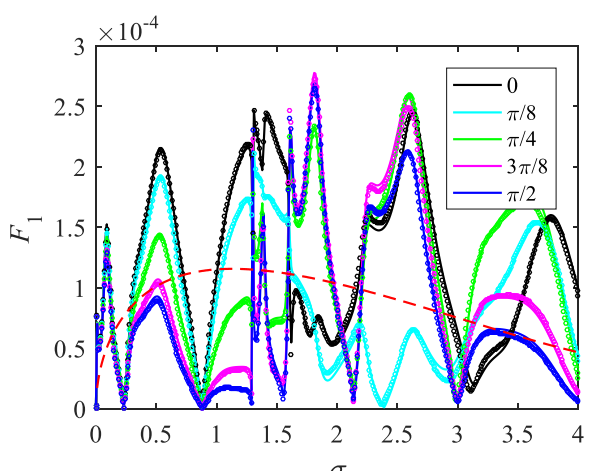

(a)

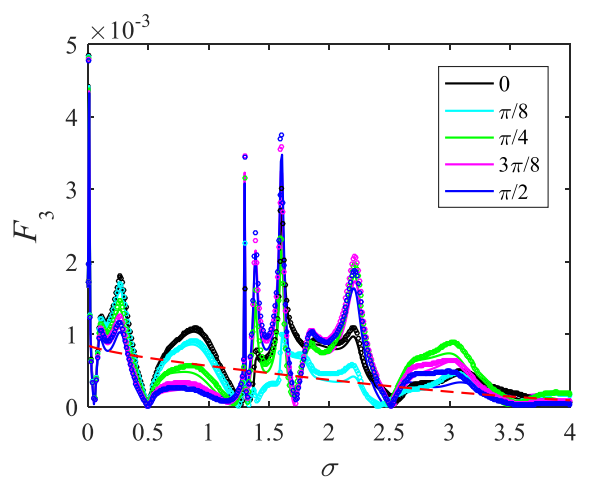

(c)

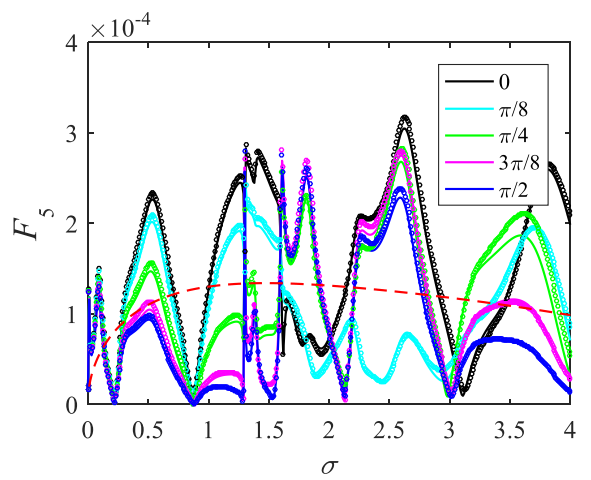

(e)

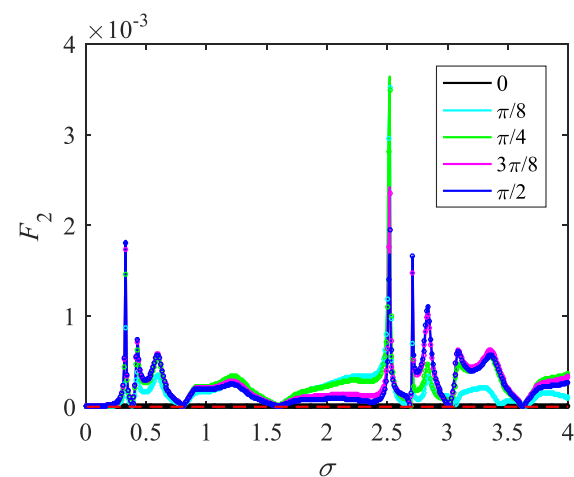

(b)

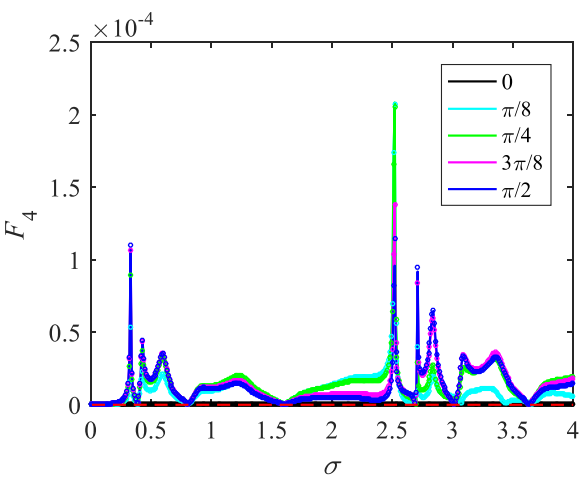

(d)

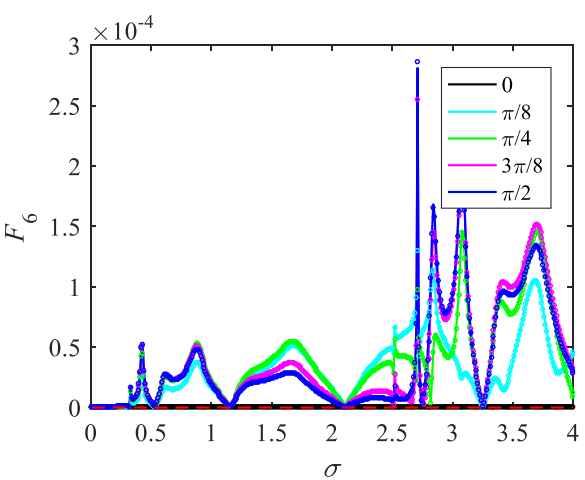

$(f)$

Figure 9 The same as Figure 7 but for wave excitation forces: $(a)$ surge; $(b)$ sway; $(c)$ heave; $(d)$ roll; $(e)$ pitch; $(f)$ yaw. Solid line: open harbour; open circles: open harbour with far field method; dashed line: in open water without 
harbour

Because of port and starboard symmetry, Eq. (26) for coupled six degrees of freedom can be decoupled into three coupled symmetric modes and three anti-symmetric modes. The amplitudes of ship motions at different wave direction $(\beta \geq 0)$ are shown in Figure 10, where $H_{i}=\left|\eta_{i} / \varsigma_{a}\right|$, $i=1, \ldots, 6$. The result in the open water at $\beta=0$ is provided for reference. From the figure, we may see that the motion response is characterized by the oscillatory behavior, which is absent in the open water. This behaviour is evidently linked to the natural modes of the harbour. The effect of natural nodes on the motion is through hydrodynamic coefficients and the excitation force. The former will mainly affect the denominator of the expression for the motion. In particular, because of the change of the added mass, the natural frequency of the ship itself, at which the restoring term cancels the inertial term, will change. The locations of motion peaks will be influenced by the combination of these factors. However, at some frequencies when the exciting force at a particular mode is virtually zero, the ship motion at that mode is expected to be small.

When $\sigma \rightarrow 0$, the motion responses of heave, roll and pitch motion mode can be found from

$$
H_{3}=\left|\frac{C_{55} f_{3}-C_{35} f_{5}}{C_{33} C_{55}-C_{53} C_{35}}\right|, H_{4}=\left|\frac{f_{4}}{C_{44}}\right|, \quad H_{5}=\left|\frac{C_{33} f_{5}-C_{53} f_{3}}{C_{33} C_{55}-C_{53} C_{35}}\right|,
$$

As $x_{g}=x_{B}$ because it is assumed that the density of the ship is uniform, Eq. (39) gives

$$
f_{3}=2 C_{33} \zeta_{a}, f_{4}=0, f_{5}=2 C_{35} \zeta_{a},
$$

which leads to $H_{3}=2, H_{4}=0$ and $H_{5}=0$, as reflected in Figure 10 .

In Figure 7 Figure 10, we may also find a common feature that the positions of large peaks are not sensitive to the wave direction. It is around e.g. $\sigma=1.3$ for surge, heave and pitch, $\sigma=0.33$ and $\sigma=2.74$ for sway, roll and yaw. We may notice that these frequencies correspond to those in Figure 7 and Figure 8 for hydrodynamic coefficients. In other words, these peaks are due to the resonant motion of the wave in the harbour, or the excitation itself has resonant feature, which leads to the large motion of the ship. 


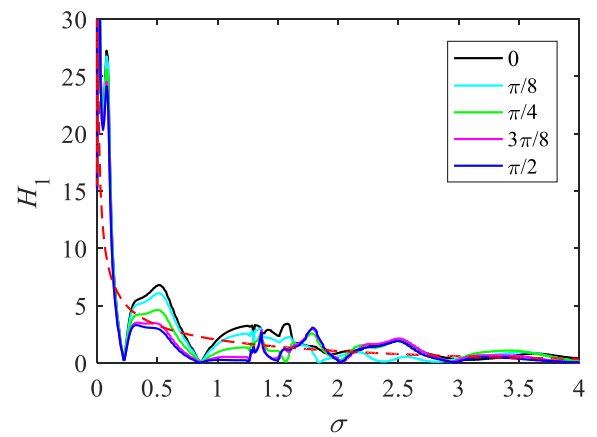

(a)

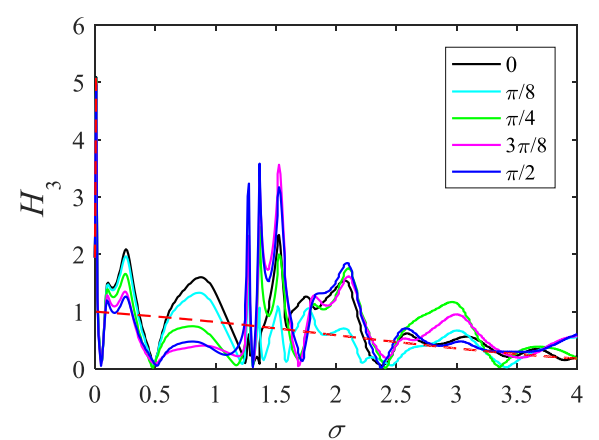

(c)

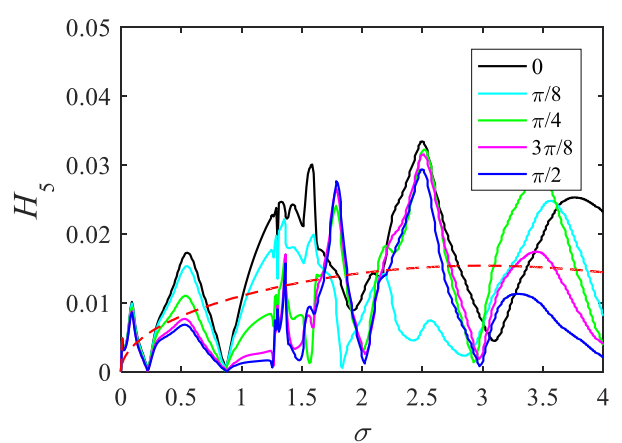

(e)

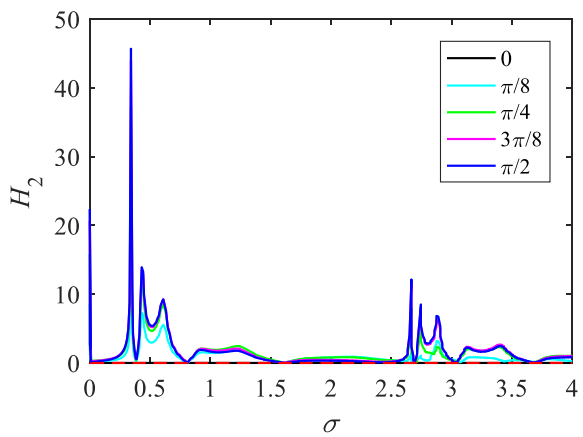

(b)

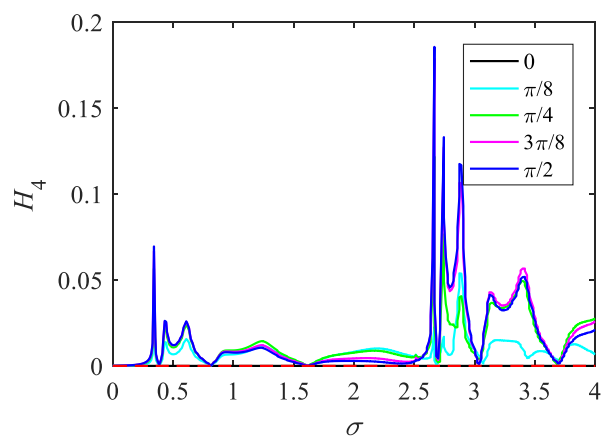

(d)

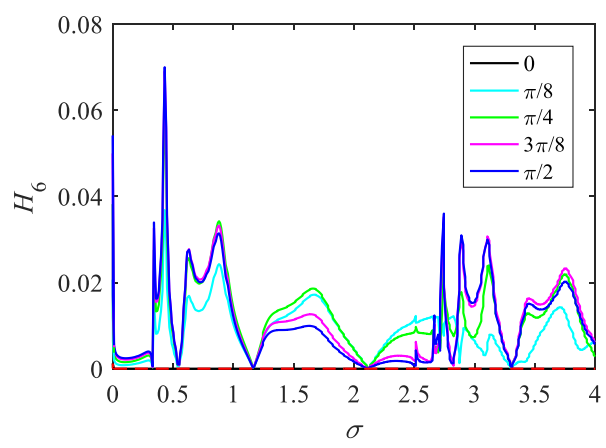

(f)

Figure 10 The same as Figure 7 but for ship motions: $(a)$ surge; $(b)$ sway; $(c)$ heave; $(d)$ roll; $(e)$ pitch; $(f)$ yaw. Solid line: in the harbour ( $\beta \in[0, \pi / 2])$; dashed line: in open water $(\beta=0)$

\subsection{The effect of ship location}

To investigate the effect of ship location, the ship is first moved longitudinally in the harbour, while its orientation remains the same. Simulations are made with the centre of gravity at $\left(-L_{s}, 0\right.$, $\left.z_{g}\right),\left(-2.5 L_{s}, 0, z_{g}\right)$ and $\left(-4 L_{s}, 0, z_{g}\right)$. It should be noted that when it is nondimensionalized, $L_{s}=1$ and $B_{s} / L_{s}=0.204$, while $z_{g}$ remains to be the same as that in Table 1 . The same number of panels as that in the previous section is used. The incident wave angle $\beta$ is taken as $\pi / 4$.

Table 3 natural frequencies of translational modes at different $x_{g}$

\begin{tabular}{ccccccccc}
\hline & Surge & \multicolumn{3}{c}{ Sway } & \multicolumn{3}{c}{ heave } \\
$x_{g}=-2.5 L_{s}$ & $x_{g}=-L_{s}$ & $x_{g}=-4 L_{s}$ & $x_{g}=-2.5 L_{s}$ & $x_{g}=-L_{s}$ & $x_{g}=-4 L_{s}$ & $x_{g}=-2.5 L_{s}$ & $x_{g}=-L_{s}$ & $x_{g}=-4 L_{s}$ \\
0.05 & 0.05 & 0.05 & $/$ & 0.22 & 0.22 & 0.22 & 0.22 & 0.22 \\
$/$ & 0.38 & 0.38 & 0.31 & 0.32 & 0.34 & $/$ & 0.32 & 0.34 \\
\hline
\end{tabular}




\begin{tabular}{|c|c|c|c|c|c|c|c|c|}
\hline 0.48 & 0.48 & 0.48 & 0.52 & 0.54 & 0.57 & 0.48 & 0.48 & 0.57 \\
\hline I & 0.80 & 0.80 & I & 0.87 & 0.87 & I & 0.54 & 0.87 \\
\hline 1.32 & 1.32 & 1.32 & 1.15 & 1.14 & 1.19 & 0.87 & 0.87 & 1.19 \\
\hline I & 1.60 & 1.62 & I & 1.24 & 1.29 & I & 1.14 & 1.29 \\
\hline 1.71 & 1.69 & 1.70 & I & 1.41 & 1.51 & 1.29 & 1.24 & 1.51 \\
\hline 2.43 & 2.41 & 2.43 & I & 1.85 & 1.85 & I & 1.41 & 1.85 \\
\hline 2.50 & 2.46 & 2.48 & I & 1.99 & 2.05 & 1.55 & 1.60 & 2.05 \\
\hline I & I & 2.69 & 2.11 & 2.12 & 2.19 & 1.85 & 1.85 & 2.19 \\
\hline I & 2.72 & 2.76 & 2.51 & 2.68 & 2.61 & I & 1.99 & 2.61 \\
\hline I & 3.05 & 3.02 & 2.74 & 2.88 & 2.78 & 2.13 & 2.12 & 2.78 \\
\hline 3.52 & 3.42 & 3.48 & I & 2.93 & 2.94 & 2.50 & 2.68 & 2.94 \\
\hline 1 & 3.70 & 3.65 & I & 3.09 & 3.10 & 2.96 & 2.93 & 3.10 \\
\hline 3.81 & 3.81 & 3.81 & 3.25 & 3.32 & 3.27 & 3.09 & 3.09 & 3.27 \\
\hline I & I & I & I & 3.44 & 3.36 & I & 3.32 & 3.36 \\
\hline I & I & I & I & I & 3.98 & 3.52 & 3.44 & 3.65 \\
\hline I & I & I & I & I & I & I & 3.70 & 3.81 \\
\hline I & I & I & I & I & & & 3.81 & 3.98 \\
\hline \multicolumn{9}{|c|}{ Table 4 natural frequencies of rotational modes at different $x_{g}$} \\
\hline & roll & & & Pitch & & & yaw & \\
\hline$x_{g}=-2.5 L_{s}$ & $x_{g}=-L_{s}$ & $x_{g}=-4 L_{s}$ & $x_{g}=-2.5 L_{s}$ & $x_{g}=-L_{s}$ & $x_{g}=-4 L_{s}$ & $x_{g}=-2.5 L_{s}$ & $x_{g}=-L_{s}$ & $x_{g}=-4 L_{s}$ \\
\hline / & 0.22 & 0.22 & 0.05 & 0.05 & 0.05 & 0.38 & 0.38 & 0.38 \\
\hline 0.31 & 0.32 & 0.34 & 0.22 & 0.22 & 0.22 & 0.80 & 0.80 & 0.48 \\
\hline 0.52 & 0.54 & 0.57 & 1 & 0.32 & 0.34 & 1 & 1.14 & 0.80 \\
\hline I & 0.87 & 0.87 & I & 0.38 & 0.38 & I & 1.31 & 1.31 \\
\hline 1.15 & 1.14 & 1.19 & 0.48 & 0.48 & 0.48 & 1.60 & 1.60 & 1.62 \\
\hline I & 1.24 & 1.29 & I & 0.80 & 0.57 & I & 1.69 & 1.70 \\
\hline I & 1.41 & 1.51 & 1.32 & 1.32 & 0.80 & I & 2.41 & 2.43 \\
\hline I & 1.85 & 1.85 & 1.55 & 1.60 & 1.32 & I & 2.46 & 2.48 \\
\hline I & 1.99 & 2.05 & 1.71 & 1.69 & 1.51 & 2.68 & 2.69 & 2.69 \\
\hline 2.11 & 2.12 & 2.19 & 2.13 & 2.12 & 1.62 & I & 2.72 & 2.76 \\
\hline 2.51 & 2.68 & 2.61 & I & 2.41 & 1.70 & 3.00 & 3.05 & 3.02 \\
\hline 2.74 & 2.88 & 2.78 & 2.50 & 2.46 & 2.05 & I & 3.42 & 3.48 \\
\hline I & 2.93 & 2.94 & I & 2.72 & 2.43 & 3.62 & 3.70 & 3.65 \\
\hline I & 3.09 & 3.10 & I & 2.93 & 2.48 & 3.98 & 3.81 & 3.81 \\
\hline 3.25 & 3.32 & 3.27 & 3.09 & 3.05 & 2.77 & I & I & I \\
\hline 3.32 & 3.44 & 3.36 & I & 3.32 & 3.02 & I & I & I \\
\hline 3.62 & 1 & 3.98 & 3.52 & 3.42 & 3.48 & I & I & 1 \\
\hline I & 1 & I & 1 & 3.70 & 3.65 & I & I & 1 \\
\hline I & I & I & 3.81 & 3.81 & 3.81 & I & I & I \\
\hline
\end{tabular}

The natural frequencies of the closed harbour with the ship at different longitudinal positions 
are presented in Table 3 and Table 4 for reference, which are obtained from the peak position of the diagonal terms of the added mass. We may notice that the lowest frequency of each mode is hardly affected by the location of the ship. The effect of $x_{g}$ on the high frequencies is quite obvious. Also, when $x_{g}$ is away from the centre of the harbour, some new peaks have appeared. Part of the reason is obviously due to that the configuration is less symmetric when $x_{g}$ is farther away from the centre and the effect of all the sloshing modes becomes more evident. For surge, pitch and yaw, the effect of natural frequencies for the anti-symmetric sloshing modes $(m=1,3,5 \ldots)$ may decrease while those for symmetric sloshing $(m=0,2,4 \ldots)$ may increase. For sway, heave and roll, on the contrary, the effect of natural frequencies for the symmetric sloshing modes may decrease while those for anti-symmetric sloshing may increase. Furthermore, the natural frequencies at $x_{g}=-L_{s}$ are close to those at $x_{g}=-4 L_{s}$ than those at $x_{g}=-2.5 L s$, which may be due to the fact that the former two cases have the same distance to the centre of the harbour.

The principal added mass coefficients of the FPSO in the open harbour are provided in Figure 11. The figure shows that generally when the ship is at the centre of the harbour, or $x_{g}=-2.5 L_{s}$, the result may have the largest peak of oscillations. While the ship is away from the centre, the largest peak is smaller in general but there are also more number of peaks as the frequency changes. Overall when the ship is near the harbour entrance, or $x_{g}=-L_{s}$ there are the most oscillations, especially for the surge and pitch motion modes. This is a reflection of stronger direct interactions with the external domain.

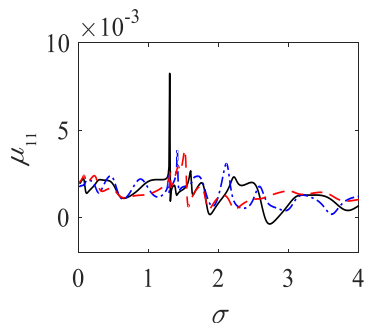

(a)

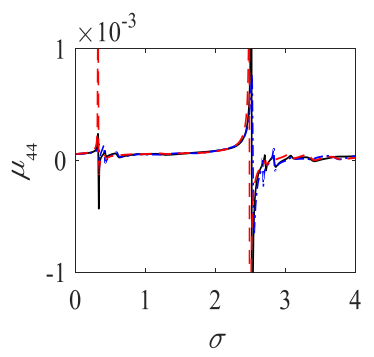

(d)

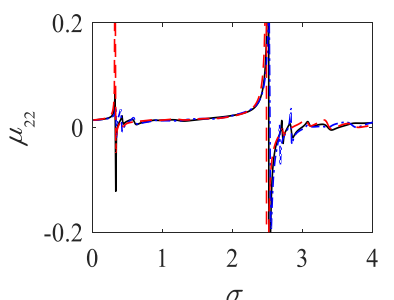

$(b)$

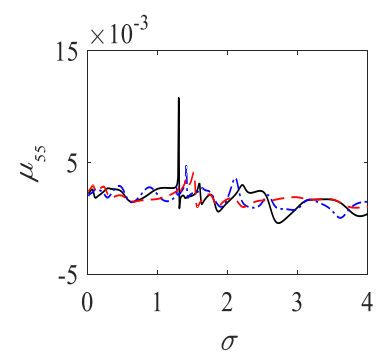

$(e)$

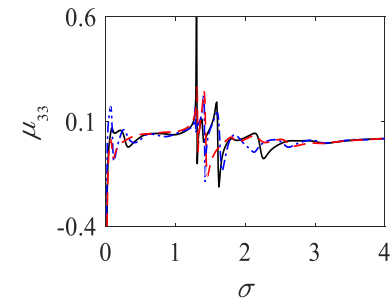

(c)

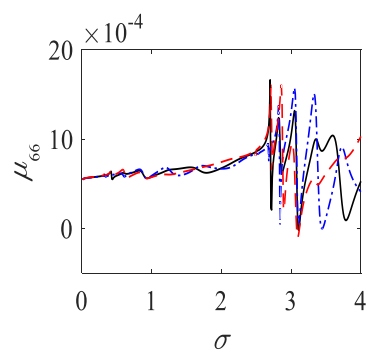

$(f)$

Figure 11 Principle added mass coefficients of the FPSO at different location in the harbour: $(a)$ surge; $(b)$ sway; $(c)$ heave; $(d)$ roll; $(e)$ pitch; $(f)$ yaw. Solid line: $\left(-2.5 L_{s}, 0, z_{g}\right)$; dashed line: $\left(-L_{s}, 0, z_{g}\right)$; dash-dotted line: $\left(-4 L_{s}, 0, z_{g}\right)$

The corresponding exciting force is shown in Figure 12. It indicates that the force is much more oscillatory when the ship is nearer to the entrance. Also at $x_{g}=-L_{s}$ there are more frequencies at 
which the force reaches a very small or close to zero. That may be explained in a sense in Figure 13, which shows the Froude-Krylov force of the FPSO next to an infinitely long coastal wall at $x=-L_{w}$. The wave includes the incident wave $\phi_{I}$ and its reflection by the wall but not the reflection by the ship. It can be seen from the figure that there are more certain frequencies where the force on the ship is located at $x_{g}=-L_{s}$ is close to zero.

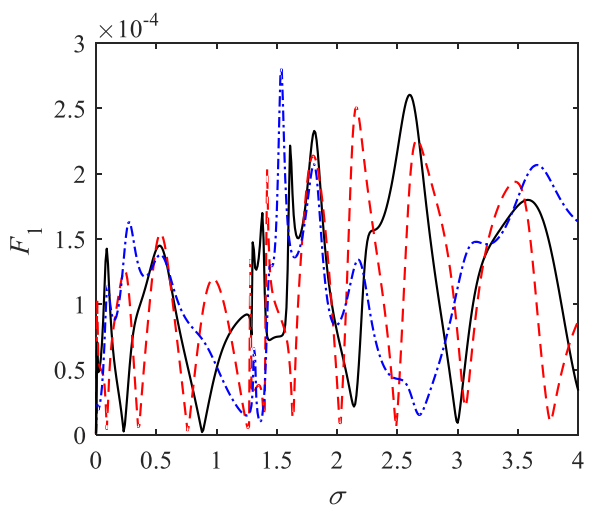

$(a)$

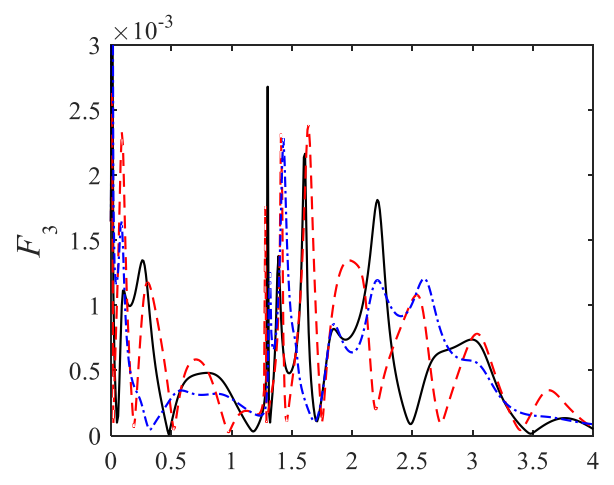

(c)

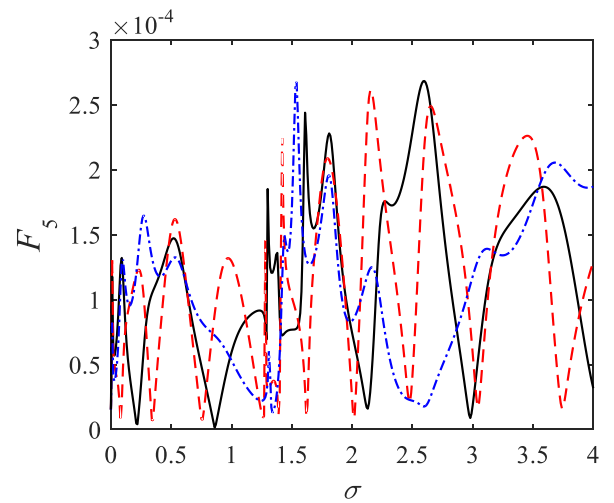

$(e)$

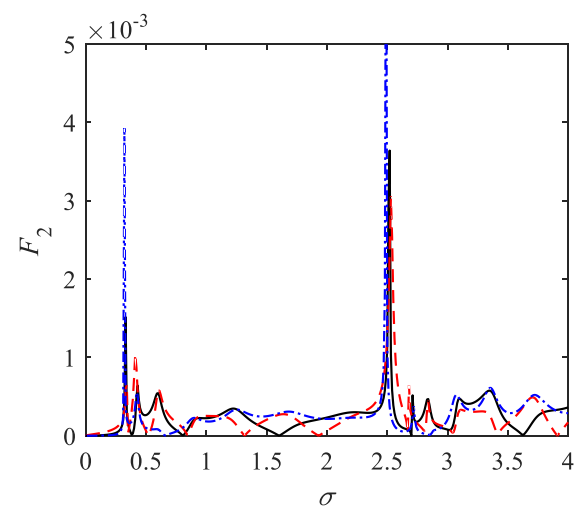

$(b)$

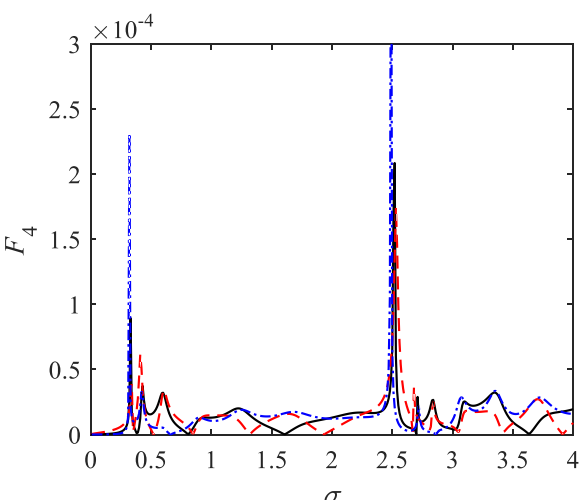

$(d)$

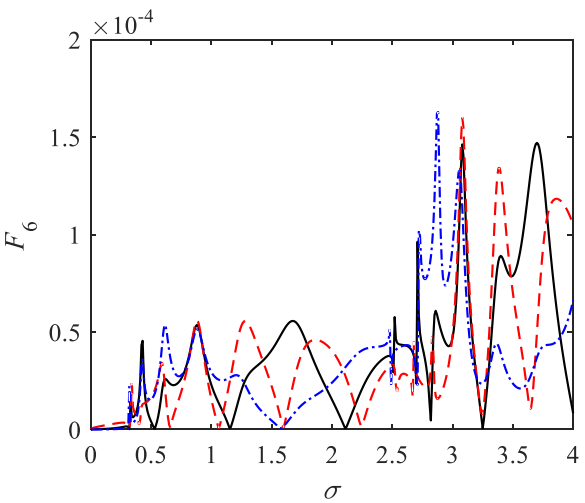

$(f)$

Figure 12 Same as Figure 11 but for wave excitation forces $(\beta=\pi / 4)$ : $(a)$ surge; $(b)$ sway; $(c)$ heave; $(d)$ roll; $(e)$ pitch; $(f)$ yaw. Solid line: $\left(-2.5 L_{s}, 0, z g\right)$; dashed line: $\left(-L_{s}, 0, z_{g}\right)$; dash-dotted line: $\left(-4 L_{s}, 0, z_{g}\right)$ 


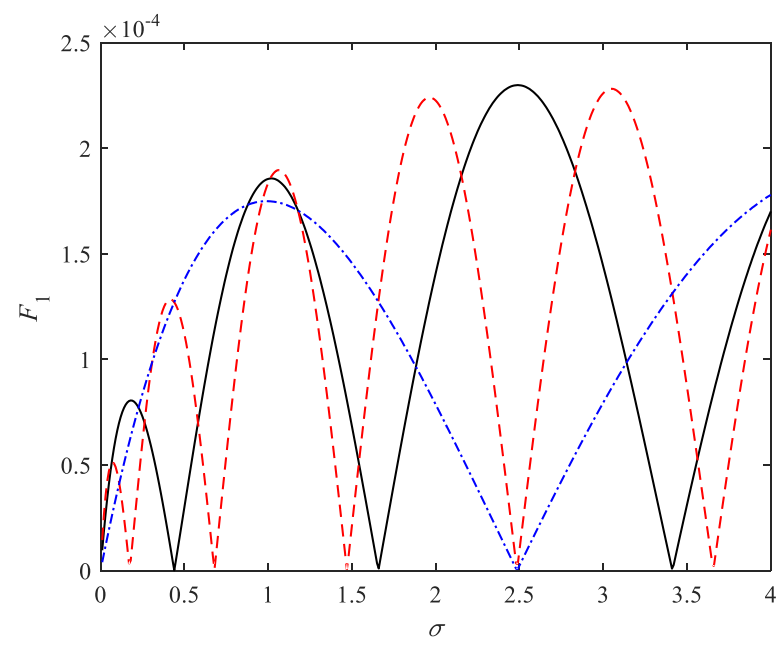

Figure 13 Surge Froude-Krylov force on FPSO next to an infinitely long wall at $x=-L_{w}$. Solid line: $\left(-2.5 L_{s}, 0, z_{g}\right)$; dashed line: $\left(-L_{s}, 0, z_{g}\right)$; dash-dotted line: $\left(-4 L_{s}, 0, z_{g}\right)$

Figure 14 gives the motion of the ship against frequency at different locations. At a given frequency, change of the location of ship can lead to significant change of its motion. This indicates that by relocating the position of the ship its wave-induced motion could be very much reduced. The locations of motion peaks are quite different at different $x_{g}$. When the motion is small or close to zero, it can be very much linked to the small or near zero exciting force in Figure 12 in the same mode. When $\sigma \rightarrow 0$, all exciting forces tend to zero or are near zero apart from $f_{3}$. However, as there is no restoring force in surge, sway and yaw, the motions in these modes do not tend to zero. This is further complicated by the first natural frequency of the closed tank $\sigma=0.05$, which is very low and corresponds to symmetric mode about $y=0$ and anti-symmetric mode about $x=-2.5 L_{s}$. Thus there is a large change in motion at the low frequency when the position of the ship changes.

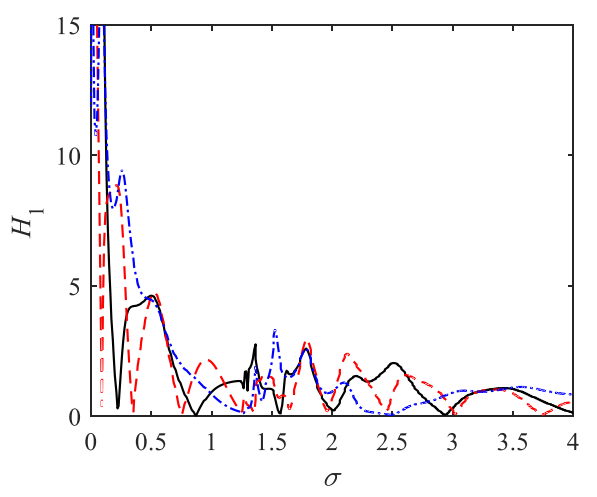

(a)

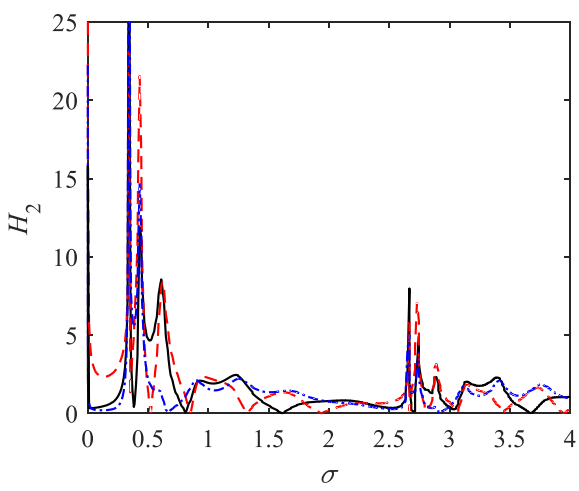

(b) 

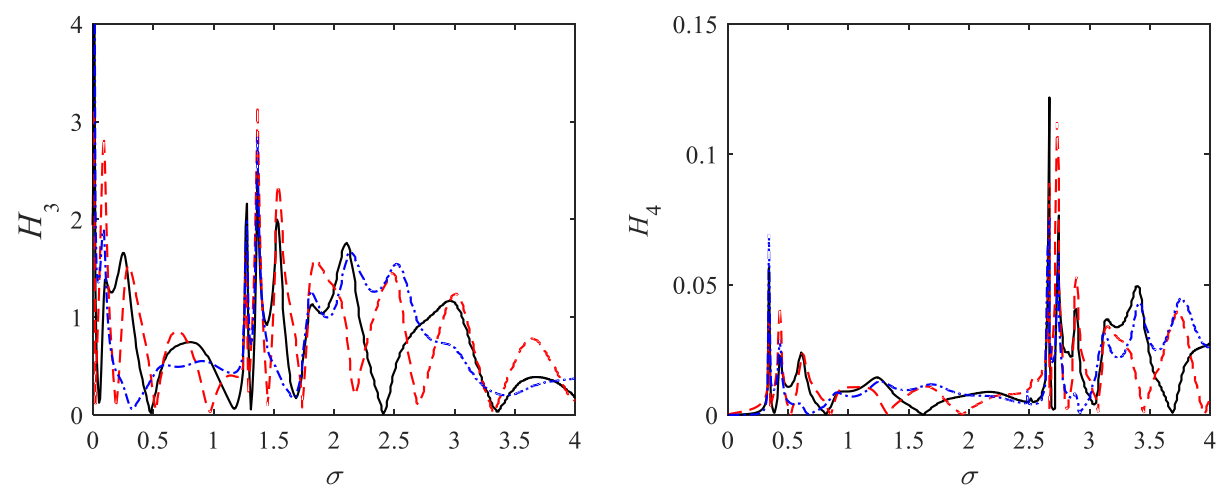

(c)

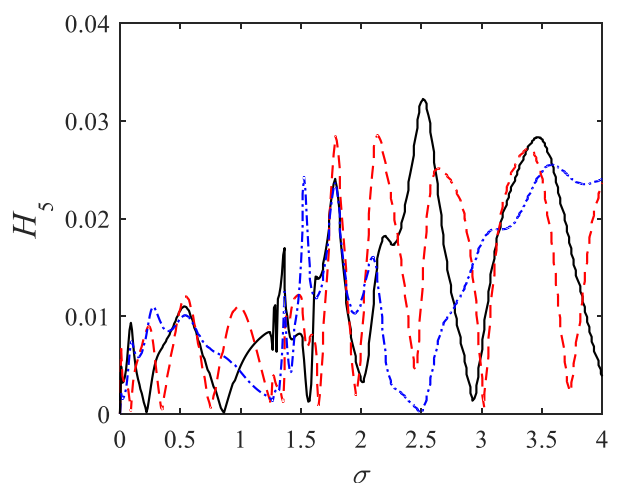

(e)

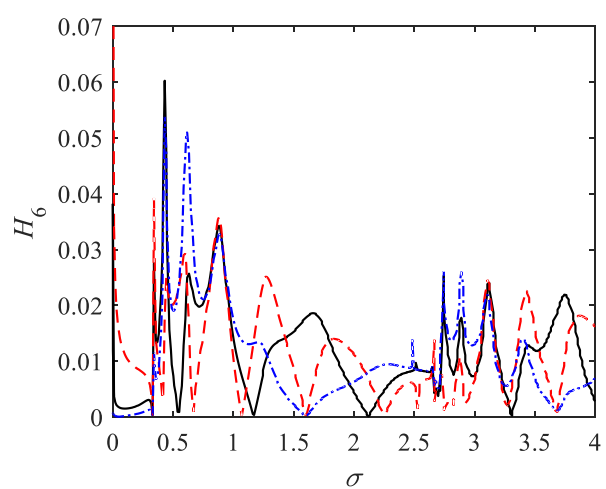

(f)

Figure 14 Same as Figure 11 but for ship motion $(\beta=\pi / 4)$ : $(a)$ surge; $(b)$ sway; $(c)$ heave; $(d)$ roll; $(e)$ pitch; $(f)$ yaw. Solid line: $\left(-2.5 L_{s}, 0, z_{g}\right)$; dashed line: $\left(-L_{s}, 0, z_{g}\right)$; dash-dotted line: $\left(-4 L_{s}, 0, z_{g}\right)$

We then undertake simulations for the ship with the different orientation angle $\gamma$, as shown in Figure 1. As the rotational centre is chosen as the gravitational centre of the ship, it will remain unchanged when the orientation of the ship changes and will remain as $\left(-2.5 L_{s}, 0, z_{g}\right)$ in the $o-x y z$ coordinate system. The wave direction $\beta$ is $\pi / 4$.

The results for ship at the harbour centre with $\gamma=0$ and $\gamma=\pi / 2$ are provided in Figure 15-Figure 16. For a ship in the open water, the hydrodynamic coefficients in each mode are not affected by its orientation. The effect of the orientation in the harbour however is highly significant. This is very much due to the natural modes of the harbour. Take $\mu_{11}$ as example, in a closed harbour it is an approximately anti-symmetric mode about $x=x_{g}$ and a symmetric mode about $y=y_{g}$ when $\gamma=0$, and it is symmetric about $x=x_{g}$ and approximately anti-symmetric about $y=y_{g}$ when $\gamma=\pi / 2$. Thus each of the natural modes of the tank corresponding to different $m$ and $n$ will have different effect. For this reason the results in Figure 15(a) and Figure 15(b) show that the positions at which $\mu_{11}$ changes sharply at $\gamma=0$ are quite close to these of $\mu_{22}$ at $\gamma=\pi / 2$, and similar links can be made between $\mu_{22}$ at $\gamma=0$ and $\mu_{11}$ at $\gamma=\pi / 2$. Similar links can be found for roll and pitch. That is reflected in Figure 15(d) and Figure 15(e). It is interesting to see that the effect of orientation of the ship on $\mu_{33}, \lambda_{33}, \mu_{66}$ and $\lambda_{66}$ are far smaller than that on the other modes. This trend also applies to the ship motion in Figure 16. The 
orientation has large effect on the surge, sway, roll and pitch. However, the effect on the heave and yaw is much smaller.

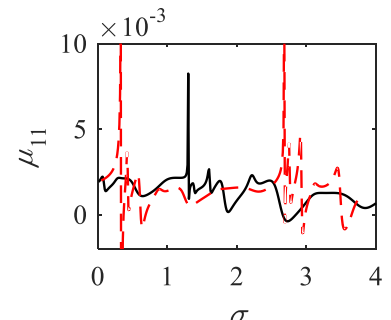

(a)

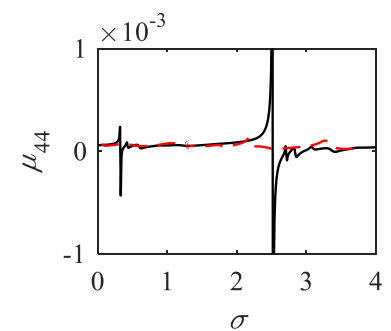

$(d)$

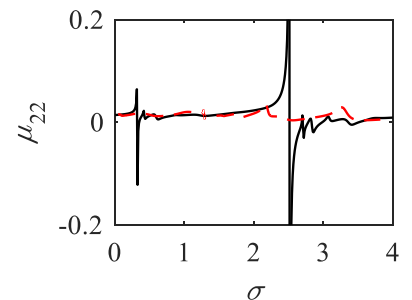

(b)

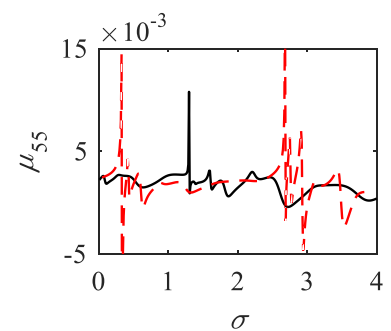

$(e)$

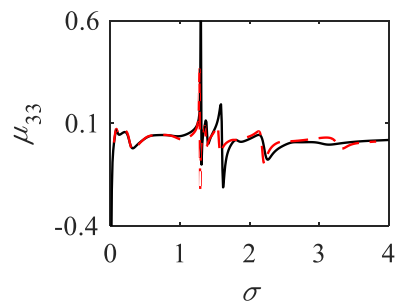

(c)

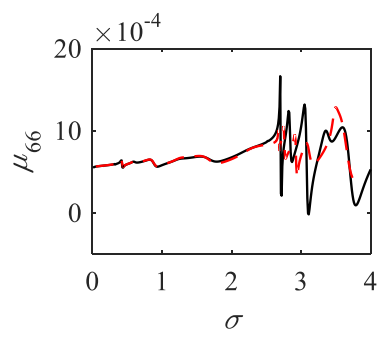

$(f)$

Figure 15 Principle added mass coefficients of the FPSO with different orientation: $(a)$ surge; $(b)$ sway; $(c)$ heave; (d) roll; (e) pitch; $(f)$ yaw. Solid line: $\gamma=0$; dashed line: $\gamma=\pi / 2$.

From Figure 16 we may see that when the ship changes its orientation into $\gamma=0$, in range of low frequency, the largest peak of surge, row and pitch is reduced sharply, comparing with that in case of orientation $\gamma=\pi / 2$. Therefore, a proper ship orientation in the harbour does help reduce the amplitude of motion.

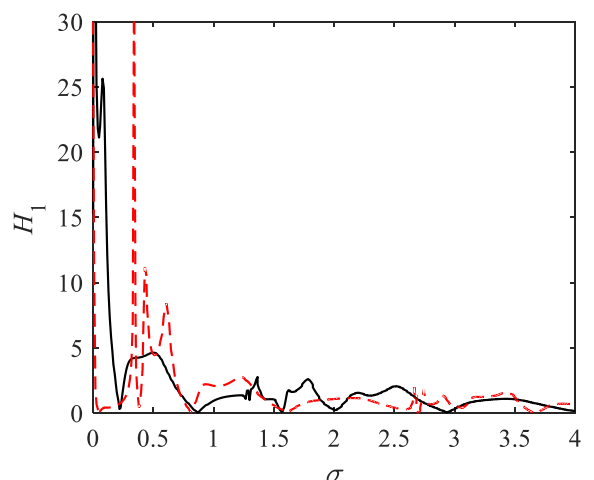

(a)

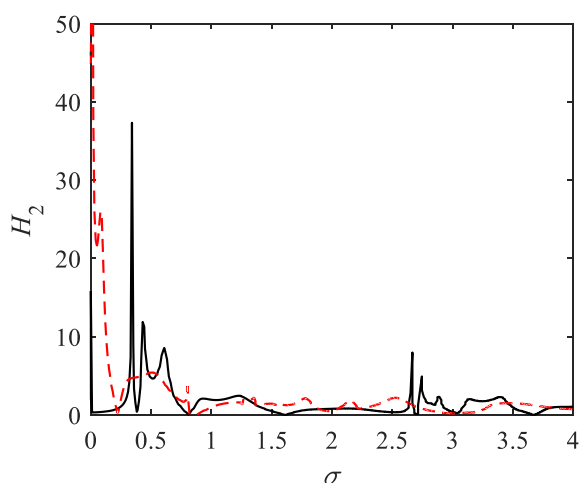

(b) 


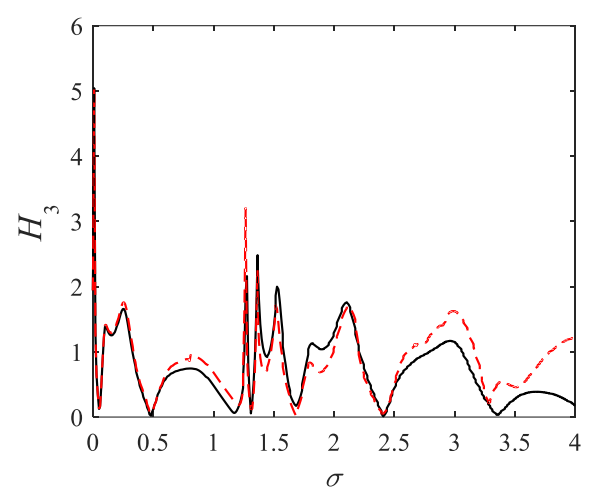

(c)

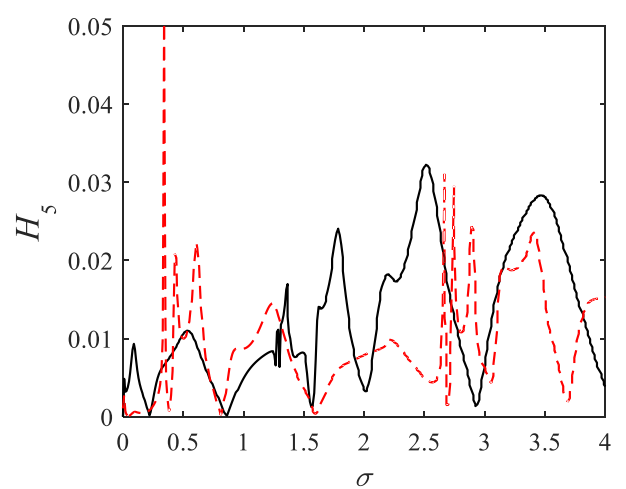

$(e)$

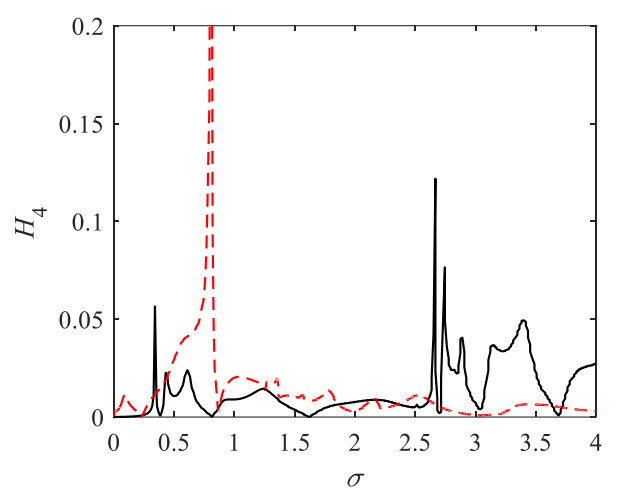

$(d)$

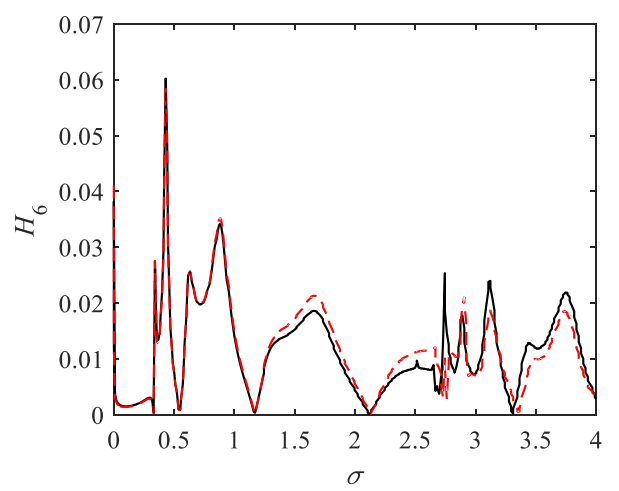

$(f)$

Figure 16 Same as Figure 15 but for ship motion $(\beta=\pi / 4)$ : $(a)$ surge; $(b)$ sway; $(c)$ heave. Solid line: $\gamma=0$; dashed line: $\gamma=\pi / 2$.

\subsection{The effect of harbour topography}

Here it may be noticed that the present approach is not limited to constant water depth. In general, for any local uneven seabed, panels can be used on the seabed in the same way as done on the harbour wall and ship hull. For the case of different water depth $h_{1}$ and $h_{2}$, inside and outside the harbour, respectively, it can be resolved by using $h_{1}$ and $h_{2}$ in Eqs. (14) and (15), respectively.

We first consider a case in which the ship floating in the centre of the harbour as in the case of Figure 7. On the bottom of the harbour right below the ship, there is a hemisphere with radius of 0.0444 and centre at $\left(-2.5 L_{w}, 0,-h\right)$. The harbour bottom shall affect the hydrodynamic features of the ships. Added masses are given in Figure 17 as an example. The figure shows that the first large peaks of the sway, heave and roll have all moved to the left, while the peaks for surge, pitch and yaw have all been lowed. 


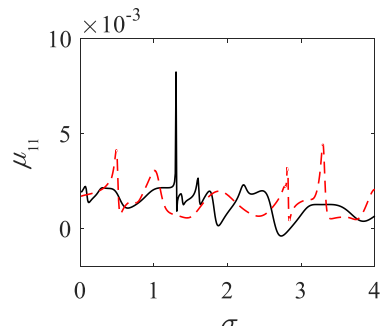

(a)

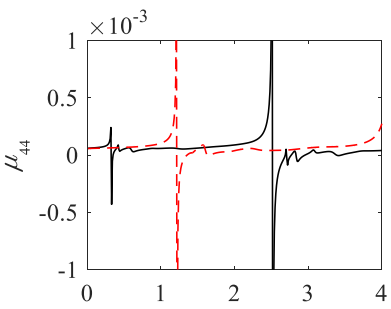

$(d)$

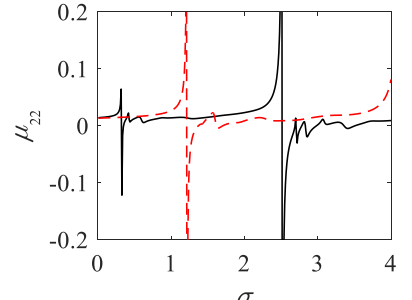

(b)

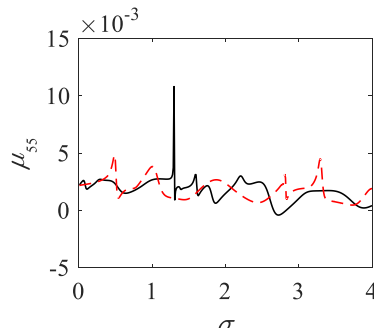

(e)

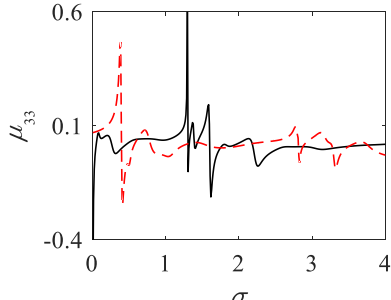

(c)

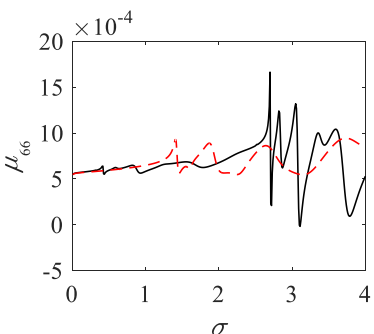

$(f)$

Figure 17 Added mass coefficients of the FPSO with a hemisphere on the harbour bottom: $(a)$ surge; $(b)$ sway; $(c)$ heave; $(d)$ roll; $(e)$ pitch; $(f)$ yaw. Solid line: flat seabed; dashed line: a hemisphere on the seabed.

We then consider the case in which depth $h_{1}$ inside the harbour is different from $h_{2}$ outside the harbour. Simulations are made for $h_{1} / h_{2}=1 / 3,2 / 3$ and 1 , respectively. Figure 18 shows the variation of the added mass with $\sigma$. From the figure we can find that the peaks are located at different places for different $\mathrm{h} 1$. This is very much due to the fact at the same frequency different h1 will correspond to a different wavenumber inside the harbour. Accordingly, the resonance in the harbour occurs at different sigma. We may replot the variation of the added mass with $k_{o} L_{s}$, and the example through surge is given in Figure 19. This shows the locations of the peaks in terms of $k_{0} L_{s}$ is not very much affected by $h_{1} / h_{2}$ varies. However, it should be noticed that at the same wavenumber, the corresponding wave frequency at different depth is different.

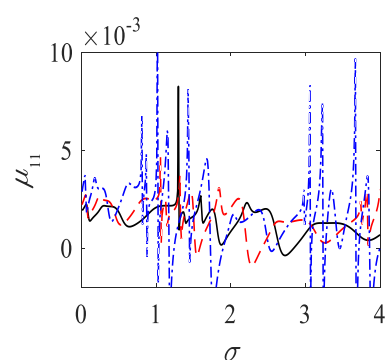

(a)

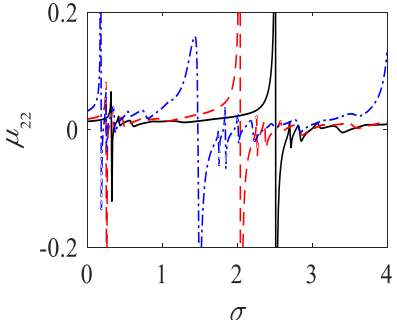

(b)

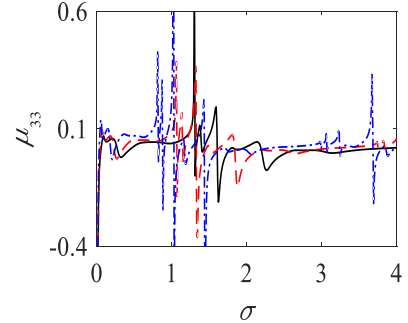

(c) 


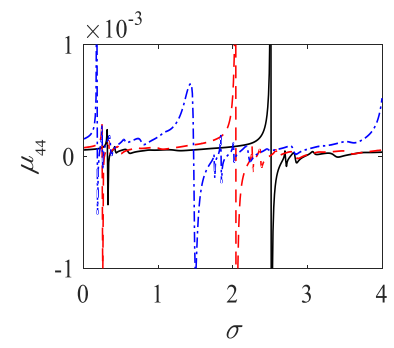

(d)

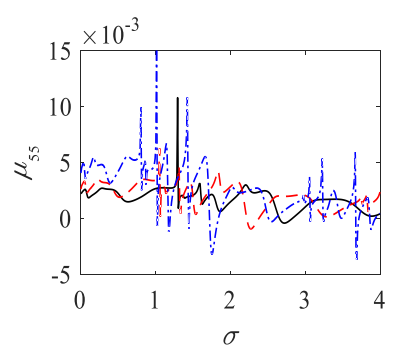

(e)

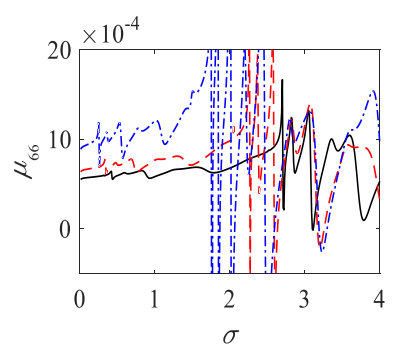

(f)

Figure 18 Added mass coefficients at different harbour water depth: (a) surge; $(b)$ sway; $(c)$ heave; $(d)$ roll; $(e)$ pitch; $(f)$ yaw. Solid line: $h_{1} / h_{2}=1$; dashed line: $h_{1} / h_{2}=2 / 3$; dash-dotted line: $h_{1} / h_{2}=1 / 3$.

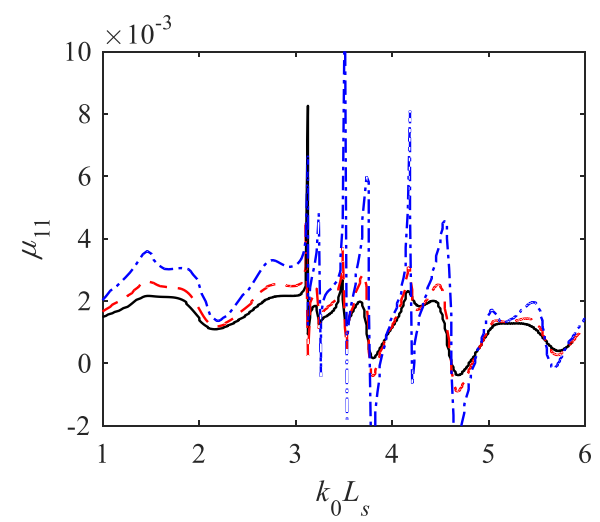

Figure 19 Surge added mass coefficients against $k_{0} L_{s}$ at different harbour depth. Solid line: $h_{1} / h_{2}=1$; dashed line: $h_{1} / h_{2}=2 / 3$; dash-dotted line: $h_{1} / h_{2}=1 / 3$.

\section{Conclusions}

A 3-D domain decomposition method has been used to investigate the wave interaction with a ship floating inside a harbour. The problem has been solved based on the linearized velocity potential theory together with the boundary element method. The effectiveness and accuracy of the numerical method has been verified through comparison with the published data. Extensive simulations have been made. From the obtained results following conclusions can be drawn:

(1) The comparison of results with existing data for a rectangular barge shows that the decomposition method is an accurate and efficient one.

(2) The hydrodynamic force and motion of a ship inside a harbour are highly oscillatory with respect to the wave frequency. The peaks and troughs of added mass and damping coefficients are very much linked to the natural frequencies of the closed harbour. The exciting forces are similarly affected by the natural frequencies. Their oscillations are also due to the reflection of the incident wave by the coastal wall and harbour walls. Unlike in the open sea, the motion curve of a ship in the harbour against frequency has multiple peaks. Not all of them are due to resonance of the ship motion in the sense the inertial term has been cancelled by the stiffness term. Many of the peaks are related to the sloshing resonance of the harbour. 
(3) The wave direction influence on a ship in an open harbour is more complex than that in open water. For surge and pitch, the motions in oblique waves may be larger than those at head sea in certain range of frequencies. For sway and roll, the motion in quartering sea may be larger than that in the beam sea.

(4) Considering the approximate equation, when the ship is away from the centre of the harbour, the configuration is less symmetric and the effect of all the sloshing modes becomes more evident. Therefore, some new peaks have appeared. For an open harbour, when the ship near the harbour entrance, the oscillatory behaviour becomes more severe than that when it is farther away from the entrance and there are also more frequencies at which forces and motions are close to zero. Furthermore, by moving the ship in the harbour properly, it is possible to reduce the amplitude of hydrodynamic force as well as motion of the ship.

(5) The ship orientation does not significantly affect the natural frequencies of the harbour. However the effect of these natural frequencies on each motion of the ship is very different. In cases of orientation angle $\gamma=0$ and $\gamma=\pi / 2$, the sway of the latter case has a close link with surge of the former case, and the surge of the latter case has oscillations similar to that of sway of the former case. Similar pattern happens in roll and pitch as well. However the effect of ship orientation on the heave and yaw motion is quite small.

(6) When the harbour has a depth different that in the open water, the results at the same frequency may be different, but they are much closer at the same wavenumber.

\section{Acknowledgement}

This work is supported by Lloyd's Register Foundation through the joint center involving University College London, Jiangsu University of Science and Technology and Harbin Engineering University, to which the authors are most grateful. Lloyd's Register Foundation helps to protect life and property by supporting engineering-related education, public engagement, and the application of research. This work is also supported by the National Natural Science Foundation of China (Grant No.11472088).

\section{Reference}

[1] Mcnown JS. Waves and seiche in idealized ports. Gravity Waves Symposium, National Bureau of Standards. 1952; Cir.:153-64.

[2] Kravtchenko J, Mcnown JS. Seiche in rectangular ports. Quart Appl Math. 1955;13:19-26.

[3] Hwang L-S, Tuck EO. On the oscillations of harbours of arbitrary shape. J Fluid Mech. 1970;42:447-64.

[4] Lee JJ. Wave-induced oscillations in harbours of arbitrary geometry. J Fluid Mech. 1971;45:375-94.

[5] Isaacson M, Qu S. Waves in a harbour with partially reflecting boundaries. Coast Eng. 1990;14:193-214.

[6] Hamanaka KI. Open, partial reflection and incident-absorbing boundary conditions in wave analysis with a boundary integral method. Coast Eng. 1997;30:281-98.

[7] Kumar P, Zhang H, Kim KI, Shi Y, Yuen DA. Wave spectral modeling of multidirectional random waves in a harbor through combination of boundary integral of Helmholtz equation with Chebyshev point discretization. Computers \& Fluids. 2015;108:13-24. 
[8] Martins-Rivas H, Mei CC. Wave power extraction from an oscillating water column along a straight coast. Ocean Engineering. 2009;36:426-33.

[9] Bellotti G. Transient response of harbours to long waves under resonance conditions. Coast Eng. 2007;54:680-93.

[10] Dong G, Gao J, Ma X, Wang G, Ma Y. Numerical study of low-frequency waves during harbor resonance. Ocean Eng. 2013;68:38-46.

[11] Guerrini M, Bellotti G, Fan Y, Franco L. Numerical modelling of long waves amplification at Marina di Carrara Harbour. Appl Ocean Res. 2014;48:322-30.

[12] Shao D, Feng X, Feng W. Numerical investigation of oscillations within a harbor of parabolic bottom induced by water surface disturbances. Appl Ocean Res. 2016;59:153-64.

[13] Sawaragi T, Kubo M. The motions of a moored ship in a harbor basin. Proc 18th Conf Coastal Engineering ASCE1982. p. 2743-62.

[14] Sawaragi T, Aoki S, Hamamoto S. Analysis of hydrodynamic forces due to waves acting on a ship in a harbour of arbitrary geometry. Proc 8th Intnl Conference Offshore Mechanics and Arctic Eng. 1989:117-23.

[15] Takagi K, Naito S, Hirota K. Hydrodynamic forces acting on a floating body in a harbor of arbitrary geometry. Proc 3th Intnl Conference Offshore and Polar Eng. 1993;4:97-104.

[16] Ohyama T, Tsuchida M. Development of a partially three-dimensional model for ship motion in a harbor with arbitrary bathymetry. 24th International Conference on Coastal Engineering. 1994;1:871-85.

[17] Ohyama T, Tsuchida M. Expanded mild-slope equations for the analysis of wave-induced ship motion in a harbor. Coast Eng. 1997;30:77-103.

[18] Kumar P, Zhang H, Kim KI, Yuen DA. Modeling wave and spectral characteristics of moored ship motion in Pohang New Harbor under the resonance conditions. Ocean Eng. 2016;119:101-13.

[19] Bingham HB. A hybrid Boussinesq-panel method for predicting the motion of a moored ship. Coast Eng. 2000;40:21-38.

[20] Wehausen JV, Laitone EV. Surface waves. Handbuch der Physik1960. p. 446-778.

[21] Hess JL, Smith AMO. Calculation of Non-lifting potential flow about arbitrary three dimensional bodies. Journal of Ship Research. 1964;8.

[22] Newman JN. Marine hydrodynamics: MIT Press; 1977.

[23] Faltinsen OM, Timokha AN. Sloshing: Cambridge University Press; 2009.

[24] Lamb H. Hydrodynamics: Cambridge University Press; 1932.

[25] Li ZF, Shi YY, Wu GX. Interaction of wave with a body floating on a wide polynya. Physics of Fluids. 2017;29:097104.

[26] Mei CC, Stiassnie M, Yue DK-P. Theory and Applications of Ocean Surface Waves: Part 1: Linear Aspects Part 2: Nonlinear Aspects: World Scientific; 1989.

[27] Mohamed HN, Yeung RW. Diffraction and radiation loads on open cylinders of thin and arbitrary shapes. J Fluid Mech. 2015;772:649-77. 